\title{
Characterization of (Molecular) Graphs with Fractional Metric Dimension as Unity
}

\author{
Muhammad Javaid (D), ${ }^{1}$ Muhammad Kamran Aslam, ${ }^{1}$ Abdulaziz Mohammed Alanazi $\left(\mathbb{D},{ }^{2}\right.$ \\ and Meshari M. Aljohani ${ }^{3}$ \\ ${ }^{1}$ Department of Mathematics, School of Science, University of Management and Technology, Lahore, Pakistan \\ ${ }^{2}$ Department of Mathematics, University of Tabuk, Tabuk, Saudi Arabia \\ ${ }^{3}$ Department of Chemistry, University of Tabuk, Tabuk, Saudi Arabia
}

Correspondence should be addressed to Muhammad Javaid; javaidmath@gmail.com

Received 5 March 2021; Accepted 11 June 2021; Published 28 June 2021

Academic Editor: Kashif Ali

Copyright (c) 2021 Muhammad Javaid et al. This is an open access article distributed under the Creative Commons Attribution License, which permits unrestricted use, distribution, and reproduction in any medium, provided the original work is properly cited.

\begin{abstract}
Distance-based dimensions provide the foreground for the identification of chemical compounds that are chemically and structurally different but show similarity in different reactions. The reason behind this similarity is the occurrence of a set $\mathbb{S}$ of atoms and their same relative distances to some ordered set $\mathbb{T}$ of atoms in both compounds. In this article, the aforementioned problem is considered as a test case for characterising the (molecular) graphs bearing the fractional metric dimension (FMD) as 1. For the illustration of the theoretical development, it is shown that the FMD of path graph is unity. Moreover, we evaluated the extremal values of fractional metric dimension of a tetrahedral diamond lattice.
\end{abstract}

\section{Introduction}

Day by day, the nexus of chemistry is progressing by the advancements in drug discovery, formation of chemical compounds, and development of testing kits for the diagnosis of different diseases and medical anomalies. Besides different concepts that arose as a result of the emergence of cheminformatics, distance-based dimensions also have their stake in this concern. Assume that, in a graph $\mathbb{C}$, the shortest path between the 2 vertices $s, t$ is given by $d(s, t)$. Let $\mathbb{S}=$ $\left\{s_{1}, s_{2}, s_{3}, \ldots, s_{k}\right\} \subseteq V(\mathbb{C})$ and $u \in V(\mathbb{C})$; then, the $k$-tuple metric form of $\mathbb{S}$ in terms of $u$ is given by $r(u \mid S)=\left(d\left(u, s_{1}\right), d\left(u, s_{2}\right), d\left(u, s_{3}\right), \ldots, d\left(u, s_{k}\right)\right)$. The set $S$ becomes a resolving set having $k$ elements for a graph $\mathbb{C}$ if each pairs of vertices in $\mathbb{C}$ bears distinct $k$-tuple metric forms. The resolving set with minimum cardinality in $\mathbb{C}$ forms its metric basis, and its cardinality represents its metric dimension.

The terminology of resolving sets was introduced by Slatter $[1,2]$ by naming them as locating sets. Harary and Melter [3] personally discovered these terminologies and called them as the metric dimension of $\mathbb{C}$. Afterward, many researchers have studied different graph structures for the calculation of metric dimensions. The results for the metric dimensions of path, cycle, Peterson, and generalized Peterson graphs can be found in [4-6]. For various results on metric dimensions of graphs, we refer to [7-9] and [10]. Chartrand et al. [11] employed metric dimension to find the solution of an integer programming problem (IPP). Subsequently, Currie and Oellermann introduced the concept of fractional metric dimension (FMD) and obtained the solution of IPP with higher accuracy [12]. Arumugam and Mathew [13] after discovering the hidden properties of FMD formally defined it. Since then, many researchers have tried their luck in this area by attacking different graph structures. The results for the FMD of graph structures as obtained from Cartesian, hierarchial, corona, lexicographic, and comb product of connected graph structures can be seen in [14-16] and $[17,18]$. Recently, Liu et al. [19] calculated the fractional metric dimension of the generalized Jahangir graph $J_{5, k}$ and Raza et al. calculated the FMD of a metal organic network $[20,21]$. Alisyah et al. presented the concept of local 
fractional metric dimension (LFMD) and found the LFMD of the corona product of two connected networks [22]. Liu et al. calculated the LFMD of rotationally symmetric and planar networks [23]. Recently, Javaid et al. calculated the bounds for the LFMD of connected and cycle-related networks in $[24,25]$.

Johnson [26, 27] employed the concept of metric dimension for creating proficiency of large datasets of chemical graph structures. The mathematical study of chemical structures concerns the development of mathematical classification of chemical compounds. The graphtheoretic version of chemical compounds naturally exists. Despite having different chemical and structural aspects, two chemical compounds show similar behaviour during the reactions. The reason behind this peculiarity is the existence of certain common substructures within these compounds. If in two compounds, the elements of the set $\mathbb{S}$ of atoms and the elements of the ordered set $\mathbb{T}$ are relatively equidistant, then we call these compounds to be similar or equivalent [28]. Finding a $\mathbb{T}$ with minimum cardinality such that the ordered lists associated with every two distinct vertices of $\mathbb{S}$ are distinct has applications to classification problems in chemistry, as described in [11].

In this article, we are going to characterise the (molecular) graphs with FMDs as unity. As a test case, we have considered the allotropic form of carbon called by tetrahedral diamond developed by Ali et al. This article propels in the following manner: Section 1 is for introduction, Section 2 is devoted for the applications of FMD in chemistry, Section 3 is for preliminaries, Section 4 concerns with the development of a tool for the characterization of graphs with FMD as 1, and Section 5 deals with the resolving neighbourhood sets of $\mathbb{D}(n)$. In Section 6, we have calculated the FMD of $\mathbb{Q D}(n)$. Section 7 gives the conclusion.

\section{Applications in Chemistry}

In a molecular graph, atoms are denoted by nodes and bond between them by edges. The fraternity of chemists and pharmacists is always in search of finding out chemical compounds in some collection bearing physiochemical properties in common at some particular places. This objective is achieved by the identification of the substructure having the smallest number of atoms. In graph theory, this problem is the same as finding the FMD of the graph under consideration. In this way, druggists and chemists will be able to capture the aforementioned features of these compounds and comprehend whether they are responsible for some pharmacological activity for a newly developed drug. For more on the applications like these, see [11].

\section{Preliminaries}

For $c \in V(\mathbb{C})$ and $\{a, b\} \subseteq V(\mathbb{C}),\{a, b\}$ is said to be resolved by $c$ if $d(a, c) \neq d(b, c)$. The set formed by the pair of nodes comprising nodes such as $c$ is called resolving neighbourhood. The resolving neighbourhood (RN) of $\{a, b\}$ is mathematically given

$R\{a, b\}=\{c \in V(\mathbb{C}) \mid d(a, c) \neq d(b, c)\}$.
Suppose a connected network $\mathbb{C}(V(\mathbb{C}), E(\mathbb{C}))$ having order $p$. A function $\tau: V(\mathbb{C}) \longrightarrow[0,1]$ is known as the resolving function (RF) of $\mathbb{C}$ if $\tau(R\{a, b\}) \geq 1 \forall a, b \in V(\mathbb{C})$, where $\tau(R\{x, y\})=\sum_{z \in R\{x, y\}} \tau(z)$. An RF $\eta$ of $\mathbb{C}$ is known as a minimal resolving function (MRF) if any function $\phi: V(\mathbb{C}) \longrightarrow[0,1]$ such that $\phi \leq \eta$ and $\phi(z) \neq \eta(z)$ for at least one $z \in V(\mathbb{C})$ that is not an RF of $\mathbb{C}$. Then, the FMD of the network $\mathbb{C}$ is given by $\operatorname{dim}_{f}(\mathbb{C})=\min \{|\eta|: \eta$ is the $M R F$ of $\mathbb{C}\}$, where $|\eta|=\sum_{z \in V(\mathbb{C})} \eta(z)[13]$.

3.1. Construction of Tetrahedral Diamond. The tetrahedral diamond graph is an $n$-dimensional lattice, comprising $n_{i}$ layers where $1 \leq i \leq n$. Figures 1 and 2 show $\mathbb{\mathbb { D }}(n)$ for $3 \leq n \leq 5$.

Each $n_{i}$ layer is having $n_{i}^{2}$ vertices, $\left(\left(n_{i}-2\right)\left(n_{i}-1\right) / 2\right)$ hexagons, and three pendent edges. The vertices of each layer are denoted by $v_{j}^{n_{i}}$ where $1 \leq j \leq n_{i}$. The first layer is isomorphic to $K_{1}$, and layer two is isomorphic to $K_{1.3}$, whereas for $1 \leq i \leq n$, each $n_{i-1}$ layer is the subgraph of the $n_{i}$-th layer. Hence, the graph formed by each layer is denoted by $S_{n_{i}}^{n_{i}}$. Similarly, following are the subgraphs found to be in all the layers: $S_{n_{j}}^{n_{i}, p}, P_{n_{i}-n_{i-1}}^{n_{i}, p}, P_{s}^{n_{i}, p}$, and $K_{1}^{n_{i}, p}$, where $1 \leq j, s \leq n_{i}-1$ and $p$ describes their position that can be top, top right, top left, bottom, bottom right, bottom left, middle, middle right, middle left, and bottom denoted by $t, t r, t l, b, b r, b l, m, m r$, and $m l$, respectively. Figure 3 shows all the subsets of $\mathbb{\square}(n)$.

It can be seen from the figure that, in each layer, $v_{1}^{n_{i}}$ is adjacent to $v_{2}^{n_{i}+1}, v_{n_{i}^{2}-n_{i-1}^{2}}^{n_{i}}$ is adjacent to $v_{n_{i}^{2}-n_{i-1}^{n_{i}+1}}$, and $v_{n_{i}^{2}}^{n_{i}^{2}}$ is adjacent to $v_{n_{i}^{2}-1}^{n_{i}+1}$. Apart from them, every vertex with an odd label in the $n_{i}-1$ layer is adjacent to the vertex with an even label in the $n_{i}$ layer and vice versa.

\section{Characterization of Graphs with FMD as Unity}

In this section of the article, we are giving generic criteria for identifying graphs with FMD as 1 . These criteria have been shaped up as a theorem given below.

Theorem 1. Let $\mathbb{C}$ be a connected graph and $R\{a, b\}$ be a resolving neighbourhood set of the pair of vertices $a, b$ in $\mathbb{C}$. If $\cap R\{a, b\} \neq \Phi$, then

$$
\operatorname{dim}_{\text {frac }}(\mathbb{C})=1,
$$

where $|V(\mathbb{C})| \geq 3$.

Proof. Assume that $R=R\{a, b\}$ is an arbitrary resolving neighbourhood set for $\{a, b\} \subset V(\mathbb{C})$ and $Y=\cap R$. Now, we define the function $\psi: V(\mathbb{C}) \longrightarrow[0,1]$ as $c=\sum_{x \in Y} \psi(x)$ and $1-c=\sum_{x \in(R-Y) \cap X} \psi(x)$, where $c$ is a real number that approaches to 1 and $X=V(\mathbb{C})$. For $a, b \in V(\mathbb{C})$ and $c \longrightarrow 1$,

$$
\begin{aligned}
\psi(R) & =\sum_{x \in R} \psi(x)=\sum_{x \in Y} \psi(x)+\sum_{x \in(R-Y) \cap X} \psi(x), \\
& =c+w(1-c) \\
& \geq 1
\end{aligned}
$$



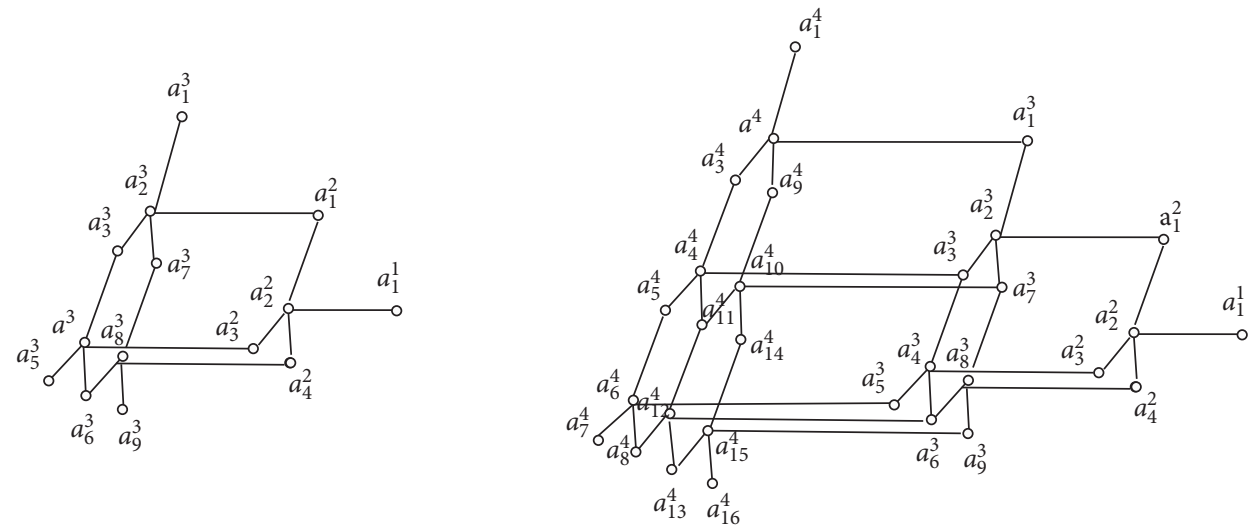

FIgure 1: Tetrahedral diamond lattice with 3 (a) and 4 (b) layers.

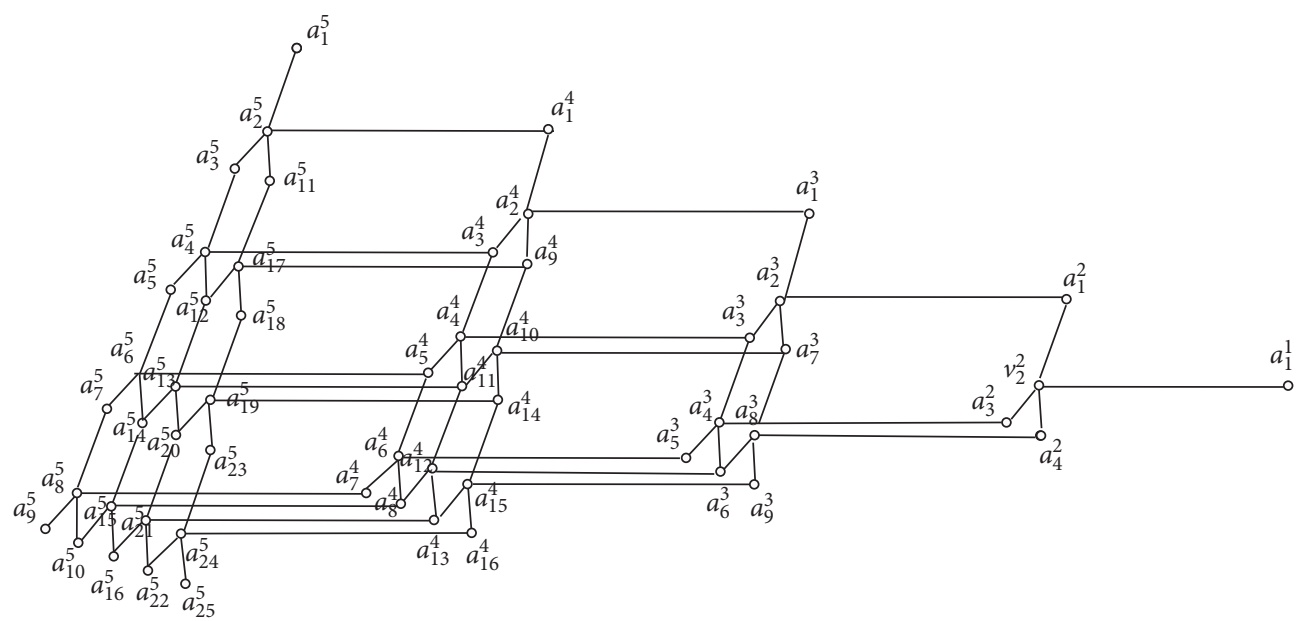

FIgURE 2: Tetrahedral diamond lattice with 5 layers.

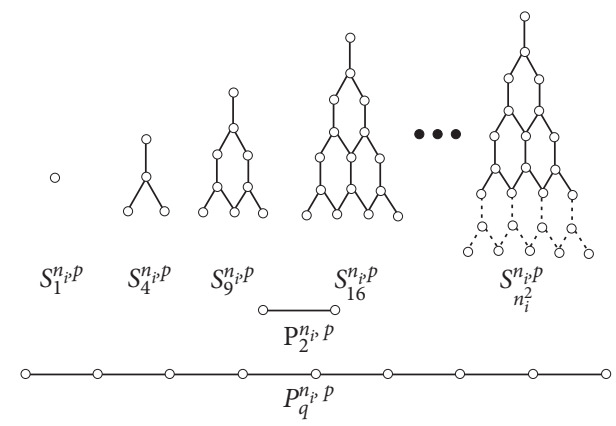

Figure 3: Subsets of the tetrahedral diamond lattice.

where $w=(|V(\mathbb{C})||V(\mathbb{C})-1| / 2)$. It implies that $\psi$ is a resolving function. To check that $\psi$ is a minimal resolving function, assume that there is another minimal resolving function $\tau$ such that $\tau \leq \psi$. By definition, $\tau(x)<\psi(x)$ for some $x \in X$. Now, for some resolving neighbourhood set $R$, we have

$$
\begin{aligned}
\tau(R) & =\sum_{x \in Y} \tau(x)+\sum_{x \in(R-Y) \cap X} \tau(x) \\
& <\sum_{x \in Y} \psi(x)+\sum_{x \in(R-Y) \cap X} \psi(x), \\
& =c+(1-c)=1 .
\end{aligned}
$$

Consequently, $\tau(R)<1$ which implies that $\tau$ is not a resolving function. Thus, $\psi$ is a minimal resolving function. Let $\bar{\psi}$ be another minimal resolving function of $\mathbb{C}$. Now, we have the following possibilities:

(i) $\bar{\psi}(x)<\psi(x) \forall x \in X$

(ii) $\bar{\psi}(x) \geq \psi(x) \forall x \in X$

(iii) $\bar{\psi}(x)<\psi(x)$ for some $x \in X$

Case 1. If $\bar{\psi}(x)<\psi(x)$ for all $x \in X$, then for each resolving neighbourhood set $R, \bar{\psi}(R)<1 \Rightarrow \bar{\psi}$ is not a resolving function; therefore, this case does not hold. 
Case 2. If $\bar{\psi}(x) \geq \psi(x)$ for all $x \in X$, then we have the following subcases:

Subcase A: for $1 \leq r, s \leq w$ and $R_{r}-Y \cap R_{s}-Y=\Phi$, we have

$$
\begin{aligned}
|\bar{\psi}| & =\sum_{x \in Y} \bar{\psi}(x)+\sum_{x \in(R-Y) \cap X} \bar{\psi}(x) \\
& >\sum_{x \in Y} \psi(x)+\sum_{x \in(R-Y) \cap X} \psi(x), \\
& =c+w(1-c)=|\psi| .
\end{aligned}
$$

As $c \longrightarrow 1, \operatorname{dim}_{\text {frac }}(\mathbb{C})=|\psi|=c+w(1-c)=1$.

Subcase B: for $1 \leq r, s \leq w$ and $R_{r}-Y \cap R_{s}-Y \neq \Phi$, we have

$$
|\bar{\psi}|=\sum_{x \in Y} \bar{\psi}(x)+\sum_{x \in \bar{R}_{t}} \bar{\psi}(x)
$$

where $\bar{R}_{t}=R_{t} \cap\left[X-\cap{ }_{j=1}^{t-1} R_{t}-Y\right]$. Then,

$$
\begin{aligned}
|\bar{\psi}| & =\sum_{x \in Y} \bar{\psi}(x)+\sum_{x \in \bar{R}_{t}} \bar{\psi}(x) \\
& \geq \sum_{x \in Y} \psi(x)+\sum_{x \in \bar{R}_{t}} \psi(x), \\
& =c+\sum_{x \in \bar{R}_{t}} \psi(x) .
\end{aligned}
$$

So,

$$
\begin{aligned}
\operatorname{dim}_{\text {frac }}(\mathbb{C}) & =c+\sum_{x \in \bar{R}_{t}} \psi(x) \\
& \leq c+\sum_{x \in(R-Y) \cap X} \psi(x), \\
& =c+w(1-c), \\
& =1 .
\end{aligned}
$$

Thus, $\operatorname{dim}_{\text {frac }}(\mathbb{C}) \leq 1$. But, by definition, $\operatorname{dim}_{\text {frac }}(\mathbb{C}) \geq 1$. Therefore,

$$
\operatorname{dim}_{\text {frac }}(\mathbb{C})=1
$$

Case 3. If $\bar{\psi}(x)<\psi(x)$ for some $x \in X$, this case is a consequent of the abovementioned two cases (Case I and II); therefore, we have $\operatorname{dim}_{\text {frac }}(\mathbb{C})=1$.

Consequently, from Case 1-3, we arrive at the following conclusion:

$$
\operatorname{dim}_{\text {frac }}(\mathbb{C})=1
$$

Using the result presented above, we are now going to prove the following fact:

Proposition 1. Suppose that, for any $n \geq 3, \mathbb{G} \cong P_{n}$; then, $\operatorname{dim}_{\text {frac }}(\mathbb{G})=1$.
Proof

Case 1: for $n=3$ : the resolving neighbourhood sets for the current case are $R_{1}=R\left\{a_{1}, a_{2}\right\}=\left\{a_{1}, a_{2}, a_{3}\right\}$, $R_{2}=R\left\{a_{2}, a_{3}\right\}=\left\{a_{1}, a_{2}, a_{3}\right\}, \quad$ and $R_{3}=$ $R\left\{a_{1}, a_{3}\right\}=\left\{a_{1}, a_{3}\right\}$. It can be seen that $\cap_{t=1}^{3} R_{t}=\left\{a_{1}, a_{3}\right\} \neq \Phi$. Therefore, from Theorem 1, we arrive at the conclusion thatdim $\operatorname{frac}_{\text {f }}\left(P_{3}\right)=1$.

Case 2: for $n \geq 4$ : the resolving neighbourhood sets of $P_{n}$ are $R\left\{a_{i}, a_{i+p}\right\}=V\left(P_{n}\right)-\left\{a_{(2 i+p / 2)}\right\} \quad$ and $R\left\{a_{i}, a_{i+s}\right\}=V\left(\mathbb{P}_{n}\right), \quad$ where $\quad p, s \geq 1, \quad 1 \leq i \leq n$, $p \equiv 0(\bmod 2)$, and $s \equiv 1(\bmod 2)$.

It can be seen that $\cap_{t=1}^{\left(\begin{array}{l}n \\ 2\end{array}\right)} R=\left\{a_{1}, a_{n}\right\} \neq \Phi$. Therefore, from Theorem 1, it implies that

$$
\operatorname{dim}_{\text {frac }}\left(P_{n}\right)=1 \text {. }
$$

Remark 1. The abovementioned proposition strengthens the result proved in [13].

\section{Resolving Neighbourhood Sets of $\mathbb{\mathbb { D }}(n)$}

In this section, we present some the results regarding the resolving neighbourhood sets of $\mathbb{\square}(n)$. Lemma 1 deals with the resolving neighbourhoods of $\mathbb{U D}(n)$ having minimum cardinality followed by Lemma 2 and Lemma 3 that are concerned with resolving neighbourhood sets of maximum cardinalities.

Lemma 1. Suppose that $\mathbb{C} \cong \mathbb{\mathbb { D }}(n)$ is an $n$-dimensional tetrahedral diamond lattice. Then, the minimum resolving neighbourhood sets are as follows:

(a) For $n \geq 4, \quad n \equiv 0(\bmod 2), \quad \alpha=(n / 2), \beta=\alpha+1$, $\gamma=2 \alpha-1, \quad$ and $\left.\eta=2 \beta-1, \quad\left|R_{t}\right|=\mid R\left\{a_{1}^{\alpha}, a_{1}^{\beta}\right\}\right\} \mid=$ $\left|R\left\{a_{\gamma}^{\alpha}, a_{\eta}^{\beta}\right\}\right|=\left|R\left\{a_{\alpha^{2}}^{\alpha}, a_{\beta^{2}}^{\beta}\right\}\right|=\left(n\left(n^{2}+3 n+2\right) / 12\right)$ and $\left|\cup_{t=1}^{3} R_{t}\right|=\left(n\left(n^{2}+3 n+2\right) / 6\right)$.

(b) For $n \geq 3, n \equiv 1(\bmod 2), \alpha=(n-1 / 2), \beta=(n+1 / 2)$, $\gamma=(n+3 / 2), \eta=2 \alpha-1, \lambda=2 \beta-1$, and $\mu=2 \gamma-1$, $\left.\left|R_{t}\right|=\mid R\left\{a_{1}^{\alpha}, a_{1}^{\beta}\right\}\right\}|=| R\left\{a_{1}^{\beta}, a_{1}^{\gamma}\right\}|=| R\left\{a_{\eta}^{\alpha}, a_{\lambda}^{\beta}\right\} \mid=$ $\left.\mid R\left\{a_{\lambda}^{\beta}, a_{\mu}^{\gamma}\right\}\right\}|=| R\left\{a_{\alpha^{2}}^{\alpha}, a_{\beta^{2}}^{\beta}\right\}|=| R\left\{a_{\beta^{2}}^{\beta}, a_{\gamma^{2}}^{\gamma}\right\} \mid=$ $\left(n^{3}+3 n^{2}+5 n+3 / 12\right)$ and $\left|\cup_{t=1}^{6} R_{t}\right|=\left(n^{3}+6 n^{2}+11 n-30 / 6\right)$.

Proof

(a) The resolving neighbourhood sets of $R\left\{a_{1}^{\alpha}, a_{1}^{\beta}\right\}, R\left\{a_{\gamma}^{\alpha}\right.$, $\left.a_{\eta}^{\beta}\right\}$, and $R\left\{a_{\alpha^{2}}^{\alpha}, a_{\beta^{2}}^{\beta}\right\}$ are $R\left\{a_{1}^{\alpha}, a_{1}^{\beta}\right\}=V(\mathbb{C})-\cup_{j=\beta}^{n}$ $\left(S_{j^{2}}^{j}-S_{(j-2)^{2}}^{j, t}\right), \quad R\left\{a_{\gamma}^{\alpha}, a_{\eta}^{\beta}\right\}=V(\mathbb{C})-\quad \cup_{j=\beta}^{n}\left(S_{j^{2}}^{j}-\right.$ $\left.S_{(j-2)^{2}}^{j, b l}\right)$ and $R\left\{a_{\gamma^{2}}^{\alpha}, a_{\eta^{2}}^{\beta}\right\}=V(\mathbb{C})-\cup_{j=\beta}^{n}\left(S_{j^{2}}^{j}-\right.$ $S_{(j-2)^{2}}^{j, b r}$. We note that $\left.\left|R_{t}\right|=\mid R\left\{a_{1}^{\alpha}, a_{1}^{\beta}\right\}\right\}|=| R\left\{a_{\gamma}^{\alpha}\right.$, $\left.a_{\eta}^{\beta}\right\}|=| R\left\{a_{(\alpha)^{2}}^{\alpha}, a_{(\alpha+1)^{2}}^{\beta}\right\} \mid=\left(n\left(n^{2}+3 n+2\right) / 12\right)$, $\cup_{t=1}^{3} R_{t}=\cup_{j=1}^{\alpha} S_{j^{2}}^{j} \cup \cup_{j=\alpha+1}^{n}\left(S_{(j-\alpha)^{2}}^{j, t} \cup S_{(j-\alpha)^{2}}^{j, b r} \cup S_{(j-\alpha)^{2}}^{j,}\right.$ $b l)$, and $\left|\cup_{t=1}^{3} R_{t}\right|=\left(n\left(n^{2}+3 n+2\right) / 6\right)$. 
(b) The resolving neighbourhood sets of $R\left\{a_{1}^{\alpha}, a_{1}^{\beta}\right\}$, $R\left\{a_{1}^{\beta}, a_{1}^{\gamma}\right\}, R\left\{a_{\eta}^{\alpha}, a_{\lambda}^{\beta}\right\}, R\left\{a_{\lambda}^{\beta}, a_{\mu}^{\gamma}\right\}, R\left\{a_{\alpha^{2}}^{\alpha}, \quad a_{\beta^{2}}^{\beta}\right\}, R\left\{a_{\beta^{2}}^{\beta}\right.$, $\left.a_{\gamma^{2}}^{\gamma}\right\} \quad$ are $\quad R\left\{a_{1}^{\alpha}, a_{1}^{\beta}\right\}=V(\mathbb{C})-\cup_{j=\beta}^{n}\left(S_{j^{2}}^{j}-S_{(j-2)^{2}}^{j, t}\right)$, $R\left\{a_{\gamma}^{\alpha}, a_{\eta}^{\beta}\right\}=V(\mathbb{C})-\cup_{j=\beta}^{n}\left(S_{j^{2}}^{j}-S_{(j-2)^{2}}^{j, b l}\right)$, and $R\left\{a_{\alpha^{2}}^{\alpha}\right.$, $\left.a_{\beta^{2}}^{\beta}\right\}=V(\mathbb{C})-\cup_{j=\beta}^{n}\left(S_{j^{2}}^{j}-S_{(j-2)^{2}}^{j, b r}\right)$ and $R\left\{a_{1}^{\beta}, a_{1}^{\gamma}\right\}=$ $V(\mathbb{C})-\cup_{j=\gamma}^{n}\left(S_{j^{2}}^{j}-S_{(j-2)^{2}}^{j, t}\right), \quad R\left\{a_{\lambda}^{\beta}, a_{\mu}^{\gamma}\right\}=V(\mathbb{C})-$ $\cup_{j=\gamma}^{n}\left(S_{j^{2}}^{j}-S_{(j-2)^{2}}^{j, b l}\right), \quad$ and $\quad R\left\{a_{\beta^{2}}^{\beta}, a_{\gamma^{2}}^{\gamma}\right\}=V(\mathbb{C})-$ $\cup_{j=\gamma}^{n}\left(S_{j^{2}}^{j}-S_{(j-2)^{2}}^{j, b r}\right)$.We note that $\left|R_{t}\right|=\mid R\left\{a_{1}^{\alpha}\right.$, $\left.\left.\left.a_{1}^{\beta}\right\}\right\}|=| R\left\{a_{1}^{\beta}, a_{1}^{\gamma}\right\}|=| R\left\{a_{\eta}^{\alpha}, a_{\lambda}^{\beta}\right\}|=| R\left\{a_{\lambda}^{\beta}, \quad a_{\mu}^{\gamma}\right\}\right\} \mid=$ $\left|R\left\{a_{\alpha^{2}}^{\alpha}, a_{\beta^{2}}^{\beta}\right\}\right|=\left|R\left\{a_{\beta^{2}}^{\beta}, a_{\gamma^{2}}^{\gamma}\right\}\right|=\left(n^{3}+3 n^{2}+\quad 5 n+\right.$ 3/12), $\cup_{t=1}^{6} R_{t}=\cup_{j=1}^{\gamma} S_{j^{2}}^{j} \cup \cup_{j=\gamma+1}^{n}\left(S_{(j-\gamma)^{2}}^{j, t} \cup \quad S_{(j-\gamma)^{2}}^{j, b r}\right.$ $\left.\cup S_{(j-\gamma)^{2}}^{j, b l}\right), \quad$ and $\quad\left|\cup_{t=1}^{6} R_{t}\right|=\left(n^{3}+6 n^{2}+\right.$ $11 n-30 / 6)$.

Lemma 2. Suppose that $\mathbb{C} \cong \mathbb{W}(n)$ is an $n$-dimensional tetrahedral diamond lattice with $n \geq 3$ and $n \equiv 1(\bmod n)$. Then,

(a) For $1 \leq \alpha \leq n, \beta=\alpha+1, \gamma=2 \alpha-1$, and $\eta=2 \beta-1$, $\left|R_{t}\right|<\left|R\left\{a_{1}^{\alpha}, a_{1}^{\beta}\right\}\right|=\left|R\left\{a_{\gamma}^{\alpha}, a_{\eta}^{\beta}\right\}\right|=\left|R\left\{a_{\alpha^{2}}^{\alpha}, a_{\beta^{2}}^{\beta}\right\}\right|$ and $\left|R\left\{a_{1}^{\alpha}, a_{1}^{\beta}\right\} \cap \cup_{t=1}^{6} R_{t}\right|=\left|R\left\{a_{\gamma}^{\alpha}, a_{\eta}^{\beta}\right\} \cap \cup_{t=1}^{6} R_{t}\right|=$ $\left|R\left\{a_{\alpha^{2}}^{\alpha}, a_{\beta^{2}}^{\beta}\right\} \cap \cup_{t=1}^{6} R_{t}\right| \geq\left|R_{t}\right|$

(b) For $1 \leq \alpha \leq n, \quad \beta=\alpha+1$ and $2 \leq \gamma \leq 2 \alpha-1$, $\left|R_{t}\right|<\left|R\left\{a_{\gamma}^{\alpha}, a_{\gamma}^{\beta}\right\}\right|$ and $\left|R\left\{a_{\gamma}^{\alpha}, a_{\gamma}^{\beta}\right\} \cup_{t=1}^{6} R_{t}\right| \leq\left|R_{t}\right|$

(c) For $2 \leq \alpha \leq n, \beta=2 \alpha-1, \gamma=2 \alpha+2, \eta=\alpha^{2}-3$, and $\mu=\alpha^{2}, \quad\left|R_{t}\right|<\left|R\left\{a_{\beta}^{\alpha}, a_{\gamma}^{\alpha}\right\}\right|=\left|R\left\{a_{\eta}^{\alpha}, a_{\mu}^{\alpha}\right\}\right|$ and $\mid R$ $\left\{a_{\beta}^{\alpha}, a_{\gamma}^{\alpha}\right\} \cup_{t=1}^{6} R_{t}|=| R\left\{a_{i}^{i}, a_{i+1}^{i+1}\right\} \cup_{t=1}^{6} R_{t} \mid=$

$\left|R\left\{a_{\eta}^{\alpha}, a_{\mu}^{\alpha}\right\} \cup_{t=1}^{6} R_{t}\right| \geq\left|R_{t}\right|$

(d) For any $\left\{a_{\alpha}^{\alpha}, a_{\beta}^{\beta}\right\} \in E(\mathbb{C}), \gamma \geq 1, \eta \geq 2, \gamma \equiv 1(\bmod 2)$, and $\quad \eta \equiv 0(\bmod 2), \quad\left|R_{t}\right|<\left|R\left\{a_{\alpha}^{\alpha}, a_{\beta}^{\beta}\right\}\right|=$ $\left|R\left\{a_{\alpha}^{\alpha}, a_{\alpha+\gamma}^{\alpha+\eta}\right\}\right|=|V(\mathbb{C})|=\left(n\left(2 n^{2}+3 n+1\right) / 6\right)$ and $\left|R\left\{a_{\alpha}^{\alpha}, a_{\beta}^{\beta}\right\} \cap \cup_{t=1}^{6} R_{t}\right| \geq\left|R_{t}\right|$

\section{Proof}

(a) The resolving neighbourhood sets of $R\left\{a_{1}^{1}, a_{1}^{2}\right\}$, $\left\{a_{1}^{1}, a_{3}^{2}\right\},\left\{a_{1}^{1}, a_{4}^{2}\right\},\left\{a_{1}^{\alpha}, a_{1}^{\beta}\right\}$, and $R\left\{a_{\gamma}^{\alpha}, a_{\eta}^{\beta}\right\}$ are $R\left\{a_{1}^{\alpha}\right.$, $\left.a_{1}^{\beta}\right\}=V(\mathbb{C})-\cup_{j=\beta}^{n}\left(S_{j^{2}}^{j}-S_{(j-1)^{2}}^{j, t}\right), \quad R\left\{a_{\gamma}^{\alpha}, a_{\eta}^{\beta}\right\}=$ $V(\mathbb{C})-\cup_{j=2}^{\beta}\left(S_{j^{2}}^{j}-S_{(j-1)^{2}}^{j, b l}\right)$, and $R\left\{a_{\alpha^{2}}^{\alpha}, a_{\beta^{2}}^{\beta}\right\}=$ $V(\mathbb{C})-\cup_{j=\beta}^{n}\left(S_{j^{2}}^{j}-S_{(j-1)^{2}}^{j, b b}\right)$, respectively.

Since $\quad\left|R\left\{a_{1}^{\alpha}, a_{1}^{\beta}\right\}\right|=\left|R\left\{a_{\gamma}^{\alpha}, a_{\eta}^{\beta}\right\}\right|=\left|R\left\{a_{\alpha^{2}}^{\alpha}, a_{\beta^{2}}^{\beta}\right\}\right|=$ $\left(2 n^{3}+3 n^{2}+n+6 \alpha(n+1)(\alpha-n)-6 \alpha \beta(\alpha-\beta-1) /\right.$ $6)$, thus $\left|R_{t}\right|=\left(2 n^{3}+3 n^{2}+n+6 \alpha(n+1)(\alpha-n)-\right.$ $6 \alpha \beta(\alpha-\beta-1) / 6)$. Therefore, $\left|R\left\{a_{1}^{\alpha}, a_{1}^{\beta}\right\} \cap \cup_{t=1}^{6} R_{t}\right|=$ $\left|R\left\{a_{\gamma}^{\alpha}, a_{\eta}^{\beta}\right\} \cap \cup_{t=1}^{6} R_{t}\right|=\left|R\left\{a_{\alpha^{2}}^{\alpha}, a_{\beta^{2}}^{\beta}\right\} \cap \cup_{t=1}^{6} R_{t}\right| \geq\left|R_{t}\right|$. (b) First of all, we introduce a notation for simplification.

$$
\mu= \begin{cases}\frac{\gamma}{2}, & \text { forn } \equiv 0(\bmod n), \\ \frac{\gamma-1}{2}, & \text { forn } \equiv 1(\bmod n) .\end{cases}
$$

The resolving neighbourhood for $R\left\{a_{\gamma}^{\alpha}, a_{\gamma}^{\beta}\right\}$ is $R\left\{a_{\gamma}^{\alpha}\right.$, $a_{\gamma}^{\beta}=V(\mathbb{C})-\cup_{j=\alpha-\mu+1}^{\alpha} S_{j^{2}}^{j} \cup \cup{ }_{j=} \beta^{n} S_{j^{2}}^{j, t} \cup \cup_{j=\mu+1}^{\mu+n-\alpha} S_{j^{2}}^{j, t}$. It can be seen that $\mid R\left\{a_{\gamma}^{\alpha}, a_{\gamma}^{\beta} \mid=\left(n-1-4(\mu+1)^{3}+\right.\right.$ $6(\mu+1)^{2}-\mu+2 b^{3}-3 b^{2}+b+2(\mu+n-\alpha+1)^{3}-$ $\left.(\mu+n-\alpha+1)^{2}-\alpha / 6\right)>\left|R_{t}\right|$. Therefore, $\mid R\left\{a_{3}^{2}, a_{4}^{2}\right\}$ $\cup_{t=1}^{6} R_{t}|\geq| R_{t} \mid$. Also, by the symmetry of the network, $\left|R_{t}\right|<\mid R\left\{a_{\gamma}^{\alpha}, a_{\gamma}^{\beta} \mid\right.$ and $\mid R\left\{a_{\gamma}^{\alpha}, a_{\gamma}^{\beta} \cup_{t=1}^{6} R_{t}|\geq| R_{t} \mid\right.$.

(c) The resolving neighbourhood for $R\left\{a_{\beta}^{\alpha}, a_{\gamma}^{\alpha}\right\}$ and $R\left\{a_{\eta}^{\alpha}, a_{\mu}^{\alpha}\right\}$ are $R\left\{a_{\beta}^{\alpha}, a_{\gamma}^{\alpha}\right\}=V(\mathbb{C})-S_{1}^{1} \cup \cup_{j=3}^{\alpha} P_{j}^{j, l} \cup$ $\cup_{j=\alpha+1}^{n}\left(P_{j}^{j, l}-S_{(j-\alpha)^{2}}^{j, l}\right)$ and $R\left\{a_{\eta}^{\alpha}, a_{\mu}^{\alpha}\right\}=V(\mathbb{C})-S_{1}^{1} \cup$ $\cup_{j=} 3^{\alpha} P_{j}^{j, r} \cup \cup_{j=\alpha+1}^{n}\left(P_{j}^{j, r}-S_{(j-\alpha)^{2}}^{j, r}\right)$. It can be seen that $\left|R\left\{a_{\beta}^{\alpha}, a_{\gamma}^{\alpha}\right\}\right|=\left|R\left\{a_{\eta}^{\alpha}, a_{\mu}{ }^{\alpha}\right\}\right|=\left(2(n+1)^{3}-3(n+1)^{2}+\right.$ $2 n+8-7 \alpha+6(2 \alpha-2)(n-\alpha+1)+2(n-\alpha+1)^{3}-$ $\left.3(n-\alpha+1)^{2} / 6\right)$. Hence, $\left|R_{t}\right|<\left(2(n+1)^{3}-3(n+\right.$ $1)^{2}+2 n+8-7 \alpha+6(2 \alpha-2)(n-\alpha+1)+2(n-\alpha+$ $\left.1)^{3}-3(n-\alpha+1)^{2} / 6\right) \quad$ and $\quad \mid R\left\{a_{\beta}^{\alpha}, a_{\gamma}^{\alpha}\right\}$ $\cup_{t=1}^{6} R_{t}|=| R\left\{a_{\eta}^{\alpha}, a_{\mu}^{\alpha}\right\} \cup_{t=1}^{6} R_{t}|\geq| R_{t} \mid$.

(d) The resolving neighbourhood sets $R\left\{a_{\alpha}^{\alpha}, a_{\beta}^{\beta}\right\}$ and $R\left\{a_{\alpha}^{\alpha}, a_{a^{\alpha+\gamma}}^{\alpha+\eta}\right\}$ are $R\left\{a_{\alpha}^{\alpha}, a_{\beta}^{\beta}\right\}=V(\mathbb{C})=R\left\{a_{\alpha}^{\alpha}, a_{a^{\alpha+\gamma}}^{\alpha+\eta}\right\}$. Clearly, $\left|R\left\{a_{\alpha}^{\alpha}, a_{\beta} \quad \beta\right\}\right|=\left|R\left\{a_{\alpha}^{\alpha}, a_{a^{\alpha+\gamma}}^{\alpha+\eta}\right\}\right|=(n(n+1)$ $(2 n+1) / 6)>\left|R_{t}\right| . \quad$ Also, $\quad\left|R\left\{a_{\alpha}^{\alpha}, a_{\beta}^{\beta}\right\} \cup \cup_{t=1}^{6} R_{t}\right|=$ $\mid R\left\{a_{\alpha}^{\alpha}, a_{a^{\alpha+\gamma}}^{\alpha+\eta} \cup_{t=1}^{6} R_{t}|\geq| R_{t} \mid\right.$.

Lemma 3. Suppose that $\mathbb{C} \cong \mathbb{W}(n)$ is an $n$-dimensional tetrahedral diamond lattice with $n \geq 4$ and $n \equiv 0(\bmod n)$. Then,

(a) For $1 \leq \alpha \leq n, \beta=\alpha+1, \gamma=2 \alpha-1$, and $\eta=2 \beta-1$, $\left|R_{t}\right|<\left|R\left\{a_{1}^{\alpha}, a_{1}^{\beta}\right\}\right|=\left|R\left\{a_{\gamma}^{\alpha}, a_{\eta}^{\beta}\right\}\right|=\left|R\left\{a_{\alpha^{2}}^{\alpha}, a_{\beta^{2}}^{\beta}\right\}\right| \quad$ and $\left|R\left\{a_{1}^{\alpha}, a_{1}^{\beta}\right\} \cap \cup_{t=1}^{3} R_{t}\right|=\left|R\left\{a_{\gamma}^{\alpha}, a_{\eta}^{\beta}\right\} \cap \cup_{t=1}^{3} R_{t}\right|=$ $\left|R\left\{a_{\alpha^{2}}^{\alpha}, a_{\beta^{2}}^{\beta}\right\} \cap \cup_{t=1}^{3} R_{t}\right| \geq\left|R_{t}\right|$

(b) For $1 \leq \alpha \leq n, \quad \beta=\alpha+1$, and $2 \leq \gamma \leq 2 \alpha-1$, $\left|R_{t}\right|<\left|R\left\{a_{\gamma}^{\alpha}, a_{\gamma}^{\beta}\right\}\right|$ and $\left|R\left\{a_{\gamma}^{\alpha}, a_{\gamma}^{\beta}\right\} \cup_{t=1}^{3} R_{t}\right| \leq\left|R_{t}\right|$

(c) For $2 \leq \alpha \leq n, \beta=2 \alpha-1, \gamma=2 \alpha+2, \eta=\alpha^{2}-3$, and $\mu=\alpha^{2}, \quad\left|R_{t}\right|<\left|R\left\{a_{\beta}^{\alpha}, a_{\gamma}^{\alpha}\right\}\right|=\left|R\left\{a_{\eta}^{\alpha}, a_{\mu}^{\alpha}\right\}\right|$ and $\mid R$ $\left\{a_{\beta}^{\alpha}, a_{\gamma}^{\alpha}\right\} \cup{ }_{t=1}^{3} R_{t}|=| R\left\{a_{i}^{i}, a_{i+1}^{i+1}\right\} \cup \cup_{t=1}^{3} R_{t} \mid=$ $\left|R\left\{a_{\eta}^{\alpha}, a_{\mu}^{\alpha}\right\} \cup_{t=1}^{3} R_{t}\right| \geq\left|R_{t}\right|$

(d) For any $\left\{a_{\alpha}^{\alpha}, a_{\beta}^{\beta}\right\} \in E(\mathbb{C}), \gamma \geq 1, \eta \geq 2, \gamma \equiv 1(\bmod 2)$, and $\quad \eta \equiv 0(\bmod 2), \quad\left|R_{t}\right|<\left|R\left\{a_{\alpha}^{\alpha}, a_{\beta}^{\beta}\right\}\right|=$ 


$$
\begin{aligned}
& \left|R\left\{a_{\alpha}^{\alpha}, a_{\alpha+\gamma}^{\alpha+\eta}\right\}\right|=|V(\mathbb{C})|=\left(n\left(2 n^{2}+3 n+1\right) / 6\right) \quad \text { and } \\
& \left|R\left\{a_{\alpha}^{\alpha}, a_{\beta}^{\beta}\right\} \cap \cup_{t=1}^{3} R_{t}\right| \geq\left|R_{t}\right|
\end{aligned}
$$

Proof. The proof is the same as that of Lemma 2.

\section{Fractional Metric Dimension of $\mathbb{\mathbb { D }}(n)$}

In this section, the FMD of $\mathbb{D D}(n)$ is calculated and the criterion of their evaluation is devised by the following result.

Theorem 2. If $\mathbb{C} \cong \mathbb{W D}(n)$ is an n-dimensional tetrahedral diamond lattice with $n \geq 3$ and $n \equiv 1(\bmod 2)$, then

$$
1<\operatorname{dim}_{\text {frac }}(\mathbb{C}) \leq 2\left(\frac{n^{3}+6 n^{2}+11 n-30}{n^{3}+3 n^{2}+5 n+3}\right) .
$$

Proof

Case 1: when $n=3$.

The resolving neighbourhood sets are as shown in Tables 1-4.

The resolving neighbourhood sets that are equal due to symmetry are given by the following:

Now, for $u v \in E(\mathbb{C})$ and $47 \leq L \leq 62$, we have

$$
R\{u v\}=V(\mathbb{C}) .
$$

In the same manner, the pairwise resolving neighbourhood sets that equals $V(\mathbb{C})$ are as follows:

As we can see, Table 3 shows the resolving neighbourhood sets of $\mathbb{U D}(3)$ having the maximum cardinality of 13 and $\cup_{L=63}^{84} R_{L}=V(\mathbb{U D}(3))$. Table 4 , on the other hand, shows the resolving neighbourhood sets with minimum cardinality of 6 and $\cup_{t=1}^{6} R_{t}=V(\mathbb{W D}(3))$. Suppose that, for $63 \leq L \leq 84$, $\left|R_{L}\right|=\gamma, \quad$ for $\quad 1 \leq t \leq 6, \quad\left|R_{t}\right|=\lambda, \quad \eta=\left|\cup_{L=63}^{84} R_{L}\right|=$ $|V(\mathbb{W}(3))|=13$, and $\delta=\left|\cup_{t=1}^{6} R_{t}\right|=|V(\mathbb{W}(3))|=13$. Now, we define a mapping $\kappa: V(\mathbb{D D}(3)) \longrightarrow[0,1]$ such that $\kappa(a)=(1 / 13)$ for all $a \in \cup_{L=63}^{84} R_{L}$; assigning the value of (1/13) to all the elements of $\cup_{L=63}^{84} R_{L}$ and summing up all the labels, we get $|\kappa|=$ $\sum_{a \in \cup_{L=63}^{84} R_{L}} \kappa(a)=(13 / 13)=1$; thus, $\operatorname{dim}_{\text {frac }}(T D)(3)=$ $|\kappa|=1$, as all the $R_{L}$ for $63 \leq L \leq 84$ are all having $V(\mathbb{D D}(3))$ in common. Similarly, we define another mapping $\tau: V(\mathbb{D D}(3)) \longrightarrow[0,1]$ such that $\tau=(1 / \lambda)=$ (1/6) for all $a \in \cup_{t=1}^{6} R_{t}$, and giving labels to the elements of $\cup_{t=1}^{6} R_{t}$ and later on summing them up, we get $|\tau|=\sum_{a \in \cup_{t=1}^{6} R_{t}} \tau(a)=(13 / 6)=2.33$; hence, $\quad \operatorname{dim}_{\text {frac }}$ $(\mathbb{C})<2.33$ as all the $R_{t}$ for $1 \leq t \leq 6$ are pairwise overlapping. Therefore, we arrive at the following conclusion:

$$
1<\operatorname{dim}_{\text {frac }}(\mathbb{W D D}(3)) \leq 2.33 .
$$

Case 2: when $n \geq 5$.

\begin{tabular}{|c|c|}
\hline $\begin{array}{l}\text { Resolving } \\
\text { neighbourhood sets }\end{array}$ & Elements \\
\hline$R_{1}=R\left\{a_{1}^{1}, a_{2}^{1}\right\}$ & $V(\mathbb{C})-\left\{a_{2}^{2}, a_{3}^{2}, a_{4}^{2}\right\} \cup\left\{a_{4}^{3}, a_{5}^{3}\right.$, \\
\hline$R_{2}=R\left\{a_{1}^{1}, a_{2}^{3}\right\}$ & $V(\mathbb{C})-\left\{a_{1}^{2}, a_{2}^{2}, a_{4}^{2}\right\}$ \\
\hline$R_{3}=R\left\{a_{1}^{1}, a_{4}^{2}\right\}$ & $V(\mathbb{C})-\left\{a_{1}^{2}, a_{2}^{2}, a_{3}^{2}\right\} \cup\left\{a_{1}^{3}, a_{2}^{3}, a_{3}^{3}, a_{4}^{3}, a_{5}^{3}\right\}$ \\
\hline$R_{4}=R\left\{a_{2}^{1}, a_{3}^{1}\right\}$ & $V(\mathbb{C})-\left\{a_{2}^{3}, a_{3}^{3}, a_{4}^{3}, a_{5}^{3}, a_{6}^{3}, a_{7}^{3}, a_{8}^{3}, a_{9}^{3}\right\}$ \\
\hline$R_{5}=R\left\{a_{3}^{2}, a_{5}^{3}\right\}$ & $V(\mathbb{C})-\left\{a_{1}^{3}, a_{2}^{3}, a_{3}^{3}, a_{4}^{3}, a_{6}^{3}, a_{7}^{3}, a_{8}^{3}, a_{9}^{3}\right\}$ \\
\hline$R_{6}=R\left\{a_{4}^{2}, a_{9}^{3}\right\}$ & $V(\mathbb{C})-\left\{a_{1}^{3}, a_{2}^{3}, a_{3}^{3}, a_{4}^{3}, a_{5}^{3}, a_{6}^{3}, a_{7}^{3}, a_{8}^{3}\right\}$ \\
\hline$R_{1}=R\left\{a_{1}^{2}, a_{3}^{2}\right\}$ & $V(\mathbb{C})-\left\{a_{1}^{1}\right\} \cup\left\{a_{2}^{2}, a_{4}^{2}\right\} \cup\left\{a_{3}^{3}, a_{8}^{3}, a_{9}^{3}\right\}$ \\
\hline$R_{2}=R\left\{a_{1}^{2}, a_{4}^{2}\right\}$ & $V(\mathbb{C})-\left\{a_{1}^{1}\right\} \cup\left\{a_{2}^{2}, a_{3}^{2}\right\} \cup\left\{a_{4}^{3}, a_{5}^{3}, a_{7}^{3}\right\}$ \\
\hline$R_{3}=R\left\{a_{1}^{2}, a_{3}^{3}\right\}$ & $V(\mathbb{C})-\left\{a_{3}^{2}\right\} \cup\left\{a_{1}^{3}, a_{2}^{3}, a_{7}^{3}, a_{8}^{3}, a_{9}^{3}\right\}$ \\
\hline$R_{4}=R\left\{a_{1}^{2}, a_{5}^{3}\right\}$ & $V(\mathbb{C})-\left\{a_{3}^{2}\right\} \cup\left\{a_{3}^{3}, a_{8}^{3}, a_{9}^{3}\right\}$ \\
\hline$R_{5}=R\left\{a_{1}^{2}, a_{6}^{3}\right\}$ & $V(\mathbb{C})-\left\{a_{3}^{2}, a_{4}^{2}\right\} \cup\left\{a_{3}^{3}, a_{7}^{3}\right\}$ \\
\hline$R_{6}=R\left\{a_{1}^{2}, a_{7}^{3}\right\}$ & $V(\mathbb{C})-\left\{a_{4}^{2}\right\} \cup\left\{a_{1}^{3}, a_{2}^{3}, a_{3}^{3}, a_{4}^{3}, a_{5}^{3}\right\}$ \\
\hline$R_{7}=R\left\{a_{1}^{2}, a_{9}^{3}\right\}$ & $V(\mathbb{C})-\left\{a_{4}^{2}\right\} \cup\left\{a_{4}^{3}, a_{5}^{3}, a_{7}^{3}\right\}$ \\
\hline$R_{8}=R\left\{a_{2}^{2}, a_{2}^{3}\right\}$ & $V(\mathbb{C})-\left\{a_{1}^{2}\right\} \cup\left\{a_{1}^{3}, a_{2}^{3}, a_{3}^{3}, a_{7}^{3}\right\}$ \\
\hline$R_{9}=R\left\{a_{2}^{2}, a_{4}^{3}\right\}$ & $V(\mathbb{C})-\left\{a_{3}^{2}\right\}-\left\{a_{1}^{3}, a_{2}^{3}, a_{7}^{3}, a_{8}^{3}, a_{9}^{3}\right\}$ \\
\hline$R_{10}=R\left\{a_{2}^{2}, a_{8}^{3}\right\}$ & $V(\mathbb{C})-\left\{a_{4}^{2}\right\} \cup\left\{a_{1}^{3}, a_{2}^{3}, a_{3}^{3}\right.$ \\
\hline$R_{11}=R\left\{a_{3}^{2}, a_{1}^{3}\right\}$ & $V(\mathbb{C})-\left\{a_{1}^{2}\right\} \cup\left\{a_{3}^{3}, a_{8}^{3}, a_{9}^{3}\right\}$ \\
\hline$R_{12}=R\left\{a_{3}^{2}, a_{3}^{3}\right\}$ & $V(\mathbb{C})-\left\{a_{1}^{2}\right\} \cup\left\{a_{4}^{3}, a_{5}^{3}, a_{6}^{3}, a_{8}^{3}, a_{9}^{3}\right\}$ \\
\hline$R_{13}=R\left\{a_{3}^{2}, a_{5}^{3}\right\}$ & $V(\mathbb{C})-\left\{a_{1}^{3}, a_{2}^{3}, a_{3}^{3}, a_{4}^{3}, a_{6}^{3}, a_{7}^{3}, a_{8}^{3}, a_{9}^{3}\right\}$ \\
\hline$R_{14}=R\left\{a_{3}^{2}, a_{6}^{3}\right\}$ & $V(\mathbb{C})-\left\{a_{4}^{2}\right\} \cup\left\{a_{1}^{3}, a_{2}^{3}, a_{3}^{3}, a_{4}^{3}, a_{5}^{3}\right\}$ \\
\hline$R_{15}=R\left\{a_{3}^{2}, a_{7}^{3}\right\}$ & $V(\mathbb{C})-\left\{a_{4}^{2}\right\} \cup\left\{a_{3}^{3}, a_{6}^{3}\right\}$ \\
\hline$R_{16}=R\left\{a_{3}^{2}, a_{9}^{3}\right\}$ & $V(\mathbb{C})-\left\{a_{4}^{2}\right\} \cup\left\{a_{4}^{3}, a_{5}^{3}, a_{7}^{3}\right\}$ \\
\hline$R_{17}=R\left\{a_{4}^{2}, a_{1}^{3}\right\}$ & $V(\mathbb{C})-\left\{a_{1}^{2}\right\} \cup\left\{a_{4}^{3}, a_{5}^{3}, a_{7}^{3}\right\}$ \\
\hline$R_{18}=R\left\{a_{4}^{2}, a_{3}^{3}\right\}$ & $V(\mathbb{C})-\left\{a_{1}^{2}, a_{3}^{2}\right\} \cup\left\{a_{6}^{3}, a_{7}^{3}, a_{7}^{3}\right\}$ \\
\hline$R_{19}=R\left\{a_{4}^{2}, a_{5}^{3}\right\}$ & $V(\mathbb{C})-\left\{a_{3}^{2}\right\} \cup\left\{a_{1}^{3}, a_{2}^{3}, a_{6}^{3}\right\}$ \\
\hline$R_{20}=R\left\{a_{4}^{2}, a_{6}^{3}\right\}$ & $V(\mathbb{C})-\cup\left\{a_{1}^{3}, a_{2}^{3}, a_{8}^{3}, a_{9}^{3}\right\}$ \\
\hline$R_{21}=R\left\{a_{4}^{\frac{1}{2}}, a_{7}^{3}\right\}$ & $V(\mathbb{C})-\left\{a_{1}^{2}\right\} \cup\left\{a_{4}^{3}, a_{5}^{3}, a_{6}^{3}, c\right.$ \\
\hline$R_{22}=R\left\{a_{1}^{1}, a_{6}^{3}\right\}$ & $V(\mathbb{C})-\left\{a_{3}^{2}, a_{4}^{2}\right\} \cup\left\{a_{1}^{3}, a_{2}^{3}\right\}$ \\
\hline$R_{23}=R\left\{a_{1}^{1}, a_{7}^{3}\right\}$ & $V(\mathbb{C})-\left\{a_{1}^{2}, a_{4}^{2}\right\} \cup\left\{a_{4}^{3}, a_{5}^{3}\right\}$ \\
\hline$R_{24}=R\left\{a_{3}^{2}, a_{4}^{2}\right\}$ & $V(\mathbb{C})-\left\{a_{1}^{1}\right\} \cup\left\{a_{1}^{2}, a_{2}^{2}\right\} \cup\left\{a_{1}^{3}, a_{2}^{3}, a_{6}^{3}\right\}$ \\
\hline$R_{25}=R\left\{a_{3}^{3}, a_{9}^{3}\right\}$ & $V(\mathbb{C})-\left\{a_{1}^{1}\right\} \cup\left\{a_{2}^{2}\right\} \cup\left\{a_{6}^{3}, a_{7}^{3}\right\}$ \\
\hline$R_{26}=R\left\{a_{4}^{3}, a_{7}^{3}\right\}$ & $V(\mathbb{C})-\left\{a_{1}^{1}\right\} \cup\left\{a_{2}^{2}\right\}$ \\
\hline$R_{27}=R\left\{a_{5}^{3}, a_{6}^{3}\right\}$ & $V(\mathbb{C})-\left\{a_{1}^{1}\right\} \cup\left\{a_{1}^{2}, a_{2}^{2}, a_{3}^{2}\right\} \cup\left\{a_{1}^{3}, a_{2}^{3}, a_{3}^{3}, a_{4}^{3}\right\}$ \\
\hline$R_{28}=R\left\{a_{6}^{3}, a_{9}^{3}\right\}$ & $V(\mathbb{C})-\left\{a_{1}^{1}\right\} \cup\left\{a_{1}^{2}, a_{2}^{2}, a_{4}^{2}\right\}$ \\
\hline$R_{29}=R\left\{a_{1}^{2}, a_{3}^{2}\right\}$ & $V(\mathbb{C})-\left\{a_{1}^{1}\right\} \cup\left\{a_{2}^{2}, a_{4}^{2}\right\} \cup\left\{a_{3}^{3}, a_{8}^{3}, a_{9}^{3}\right\}$ \\
\hline$R_{30}=R\left\{a_{1}^{2}, a_{4}^{2}\right\}$ & $V(\mathbb{C})-\left\{a_{1}^{1}\right\} \cup\left\{a_{2}^{2}, a_{3}^{2}\right\} \cup\left\{a_{4}^{3}, a_{5}^{3}, a_{7}^{3}\right\}$ \\
\hline$R_{31}=R\left\{a_{1}^{\frac{1}{3}}, a_{3}^{3}\right\}$ & $V(\mathbb{C})-\left\{a_{1}^{1}\right\} \cup\left\{a_{1}^{2}, a_{2}^{2}, a_{4}^{2}\right\} \cup\left\{a_{2}^{3}, a_{7}^{3}, a_{8}^{3}\right\}$ \\
\hline$R_{32}=R\left\{a_{1}^{3}, a_{6}^{3}\right\}$ & $V(\mathbb{C})-\left\{a_{1}^{1}\right\} \cup\left\{a_{2}^{2}\right\} \cup\left\{a_{3}^{3}, a_{7}^{3}\right\}$ \\
\hline$R_{43}=R\left\{a_{1}^{3}, a_{7}^{3}\right\}$ & $V(\mathbb{C})-\left\{a_{1}^{1}\right\} \cup\left\{a_{1}^{2}, a_{2}^{2}, a_{3}^{2}\right\} \cup\left\{a_{2}^{3}, a_{3}^{3}, a_{4}^{3}, a_{5}^{3}\right\}$ \\
\hline$R_{33}=R\left\{a_{2}^{\frac{1}{3}}, a_{4}^{3}\right\}$ & $V(\mathbb{C})-\left\{a_{1}^{1}\right\} \cup\left\{a_{2}^{2}, a_{4}^{2}\right\} \cup\left\{a_{3}^{3}, a_{8}^{3}, a_{9}^{3}\right\}$ \\
\hline$R_{34}=R\left\{a_{2}^{3}, a_{8}^{3}\right\}$ & $V(\mathbb{C})-\left\{a_{1}^{1}\right\} \cup\left\{a_{2}^{2}, a_{3}^{2}\right\} \cup\left\{a_{4}^{3}, a_{5}^{3}, a_{7}^{3}\right\}$ \\
\hline$R_{35}=R\left\{a_{5}^{3}, a_{7}^{3}\right\}$ & $V(\mathbb{C})-\left\{a_{1}^{1}\right\} \cup\left\{a_{2}^{2}\right\} \cup\left\{a_{3}^{3}, a_{6}^{3}\right\}$ \\
\hline
\end{tabular}

The required minimum resolving neighbourhood sets are

$$
R\left\{a_{1}^{\alpha}, a_{1}^{\beta}\right\}, R\left\{a_{1}^{\beta}, a_{1}^{\gamma}\right\}, R\left\{a_{\eta}^{\alpha}, a_{\lambda}^{\beta}\right\}, R\left\{a_{\lambda}^{\beta}, a_{\mu}^{\gamma}\right\},
$$

TABLE 1: Resolving neighbourhood sets of $\mathbb{W D}(3)$.

$R\left\{a_{\alpha^{2}}^{\alpha}, a_{\beta^{2}}^{\beta}\right\}$, and $R\left\{a_{\beta^{2}}^{\beta}, a_{\gamma^{2}}^{\gamma}\right\}$, where $\alpha=(n-1 / 2)$, $\beta=(n+1 / 2), \quad \gamma=(n+3 / 2), \quad \eta=2 \alpha-1, \quad \lambda=2 \beta-1$, and $\mu=2 \gamma-1$. Lemma 1 clarifies that $\left|R_{t}\right|=\left|R\left\{a_{1}^{\alpha}, a_{1}^{\beta}\right\}\right|=$ $\left|R\left\{a_{1}^{\beta}, a_{1}^{\gamma}\right\}\right|=\left|R\left\{a_{\eta}^{\alpha}, a_{\lambda}^{\beta}\right\}\right|=\left|R\left\{a_{\lambda}^{\beta}, a_{\mu}^{\gamma}\right\}\right|=$ $\left|R\left\{a_{\alpha^{2}}^{\alpha}, a_{\beta^{2}}^{\beta}\right\}\right|=\left|R\left\{a_{\beta^{2}}^{\beta}, a_{\gamma^{2}}^{\gamma}\right\}\right|=\left(n^{3}+3 n^{2}+5 n+\right.$ $3 / 12) \leq|R\{a, b\}|$ for all $a, b \in V(\mathbb{C})$ and $\cup_{t=1}^{6} R_{t}=\cup_{j=1}^{\gamma} S_{j^{2}}^{j} \cup \cup_{j=\gamma+1}^{n} \quad\left(S_{(j-\gamma)^{2}}^{j, t} \cup S_{(j-\gamma)^{2}}^{j, b r} \cup S_{(j-\gamma)^{2}}^{j, b l}\right)$. Also, the resolving neighbourhood sets with maximum cardinality of $|V(\mathbb{C})|$, as clarified by Lemma 2 , are $R_{1}=$ $R\left\{a_{\alpha}^{\alpha}, a_{a^{\alpha+\gamma}}^{\alpha+\eta}\right\}$ and $R_{1}=R\left\{a_{\alpha}^{\alpha}, a_{\beta}^{\beta}\right\}$ and $R_{1}=R\left\{a_{\alpha}^{\alpha}, a_{a^{\alpha+\gamma}}^{\alpha+\eta}\right\}$, respectively. Moreover, $\cup_{L=1}^{2} R_{L}=V(\mathbb{C})$. Let 
$\left|R\left\{a_{\alpha}^{\alpha}, a_{a^{\alpha+\gamma}}^{\alpha+\eta}\right\}\right|=\left|R\left\{a_{\alpha}^{\alpha}, a_{\beta}^{\beta}\right\}\right|=\gamma,\left|R_{t}\right|=\lambda,\left|\cup_{L=1}^{2} R_{L}\right|=\eta$, and $\left|\cup_{t=1}^{6} R_{t}\right|=\delta$. To find the minimum value for the $\operatorname{dim}_{\text {frac }}(\mathbb{C})$, we define a mapping $\kappa: V(\mathbb{C}) \longrightarrow[0,1]$ such that

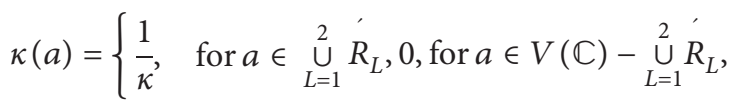

where $\gamma=\eta=\left(n\left(2 n^{2}+3 n+1\right) / 6\right)$. Assigning the labels to the elements of $\bigcup \cup{ }_{L=1}^{2} R_{L}$ and summing them up, we get $|\kappa|=\sum_{a \in \cup_{L=1}^{2} R_{L}}(1 / \gamma)=1$.

Similarly, for the maximum value of $\operatorname{dim}_{\text {frac }}(\mathbb{C})$, we define another mapping $\tau: V(\mathbb{C}) \longrightarrow[0,1]$ such that

$$
\tau(a)=\left\{\frac{1}{\lambda}, \quad \text { for } a \in \bigcup_{t=1}^{6} R_{t}, 0, \text { for } a \in V(\mathbb{C})-\bigcup_{t=1}^{6} R_{t} .\right.
$$

It can be seen that $\tau$ is a resolving function for $\mathbb{C}$ with $n \geq 3$ because $\tau(R\{u, v\}) \geq 1 \forall u, v \in V(\mathbb{C})$. On the contrary, assume that there is another resolving function $\rho$ such that $\rho(u) \leq \tau(u)$, for at least one $u \in V(\mathbb{C}) \rho(u) \neq \tau(u)$. As a consequence, $\rho(R\{u, v\})<1$, where $R\{u, v\}$ is a resolving neighbourhood of $\mathbb{C}$ with minimum cardinality $\lambda$. It shows that $\rho$ is not a resolving function which is a contradiction. Therefore, $\tau$ is a minimal resolving function that attains minimum $|\tau|$ for $\mathbb{C}$. Since all the $R_{t}$ have nonempty intersection, there is another minimal resolving function of $\bar{\tau}$ of $\mathbb{C}$ such that $|\bar{\tau}| \leq|\tau|$. Hence, assigning $(1 / \lambda)$ to the vertices of $\mathbb{C}$ in $\cup_{t=1}^{3} R_{t}$ and calculating the summation of all the weights, we get

$$
\operatorname{dim}_{\text {frac }}(\mathbb{C})=\sum_{t=1}^{\delta} \frac{1}{\lambda} \leq 2 \frac{n^{3}+6 n^{2}+11 n-30}{n^{3}+3 n^{2}+5 n+3} .
$$

In the end, we arrive at the following finding:

$$
1<\operatorname{dim}_{\text {frac }}(\mathbb{C}) \leq 2\left(\frac{n^{3}+6 n^{2}+11 n-30}{n^{3}+3 n^{2}+5 n+3}\right) .
$$

Theorem 3. If $\mathbb{C} \cong \mathbb{U} \mathbb{D}(n)$ is an $n$-dimensional tetrahedral diamond lattice with $n \geq 4$ and $n \equiv 0(\bmod 2)$, then

$$
1<\operatorname{dim}_{\text {frac }}(\mathbb{C}) \leq 2 \text {. }
$$

Proof

Case 1: when $n=4$.

The resolving neighbourhood sets are as shown in Tables 4-6.

The resolving neighbourhood sets that are equal due to symmetry are given by the following:

For $109 \leq L \leq 149$ and $\{u v\} \in V(\mathbb{C})$, we have

$$
R_{L}=R\{u v\}=V(\mathbb{C}) .
$$

Similarly, the pairwise resolving neighbourhood sets that equals $V(\mathbb{C})$ are as follows:

As we can see, Table 7 shows the resolving neighbourhood sets of $\mathbb{C}$ having the maximum cardinality of
TABLE 2: Resolving neighbourhood sets of $\mathbb{W}(3)$ equal due to symmetry.

\begin{tabular}{ll}
\hline Resolving neighbourhood sets & Elements \\
\hline$R_{36}=R\left\{a_{1}^{1}, a_{1}^{3}\right\}$ & $R\left\{a_{2}^{2}, a_{2}^{3}\right\}$ \\
$R_{38}=R\left\{a_{1}^{1}, a_{5}^{3}\right\}$ & $R\left\{a_{2}^{2}, a_{4}^{3}\right\}$ \\
$R_{40}=R\left\{a_{3}^{3}, a_{7}^{3}\right\}$ & $R\left\{a_{3}^{2}, a_{4}^{2}\right\}$ \\
$R_{42}=R\left\{a_{6}^{3}, a_{7}^{3}\right\}$ & $R\left\{a_{1}^{2}, a_{3}^{2}\right\}$ \\
$R_{44}=R\left\{a_{3}^{3}, a_{6}^{3}\right\}$ & $R\left\{a_{1}^{2}, a_{4}^{2}\right\}$ \\
$R_{46}=R\left\{a_{1}^{3}, a_{9}^{3}\right\}$ & $R\left\{a_{1}^{2}, a_{4}^{2}\right\}$ \\
$R_{37}=R\left\{a_{1}^{1}, a_{3}^{3}\right\}$ & $R\left\{a_{2}^{2}, a_{4}^{3}\right\}$ \\
$R_{39}=R\left\{a_{1}^{1}, a_{9}^{3}\right\}$ & $R\left\{a_{2}^{2}, a_{8}^{3}\right\}$, \\
$R_{41}=R\left\{a_{4}^{3}, a_{8}^{3}\right\}$ & $R\left\{a_{3}^{2}, a_{4}^{2}\right\}$ \\
$R_{43}=R\left\{a_{7}^{3}, a_{9}^{3}\right\}$ & $R\left\{a_{1}^{2}, a_{3}^{2}\right\}$ \\
$R_{45}=R\left\{a_{1}^{3}, a_{5}^{3}\right\}$ & $R\left\{a_{1}^{2}, a_{3}^{2}\right\}$ \\
\hline
\end{tabular}

TABLE 3: Resolving neighbourhood sets of $\mathbb{D}(3)$ equal to $V(\mathbb{W D}(3))$.

\begin{tabular}{lr}
\hline Resolving neighbourhood sets & Elements \\
\hline$R_{63}=R\left\{a_{1}^{1}, a_{2}^{3}\right\}$ & $V(\mathbb{C})$ \\
$R_{65}=R\left\{a_{1}^{1}, a_{8}^{3}\right\}$ & $V(\mathbb{C})$ \\
$R_{67}=R\left\{a_{1}^{2}, a_{8}^{3}\right\}$ & $V(\mathbb{C})$ \\
$R_{69}=R\left\{a_{2}^{2}, a_{3}^{3}\right\}$ & $V(\mathbb{C})$ \\
$R_{71}=R\left\{a_{2}^{2}, a_{6}^{3}\right\}$ & $V(\mathbb{C})$ \\
$R_{73}=R\left\{a_{2}^{2}, a_{9}^{3}\right\}$ & $V(\mathbb{C})$ \\
$R_{75}=R\left\{a_{3}^{2}, a_{4}^{3}\right\}$ & $V(\mathbb{C})$ \\
$R_{77}=R\left\{a_{4}^{2}, a_{2}^{3}\right\}$ & $V(\mathbb{C})$ \\
$R_{79}=R\left\{a_{4}^{2}, a_{8}^{3}\right\}$ & $V(\mathbb{C})$ \\
$R_{81}=R\left\{a_{1}^{3}, a_{8}^{3}\right\}$ & $V(\mathbb{C})$ \\
$R_{83}=R\left\{a_{2}^{3}, a_{6}^{3}\right\}$ & $V(\mathbb{C})$ \\
$R_{64}=R\left\{a_{1}^{1}, a_{4}^{3}\right\}$ & $V(\mathbb{C})$ \\
$R_{66}=R\left\{a_{1}^{2}, a_{4}^{3}\right\}$ & $V(\mathbb{C})$ \\
$R_{68}=R\left\{a_{2}^{2}, a_{1}^{3}\right\}$ & $V(\mathbb{C})$ \\
$R_{70}=R\left\{a_{2}^{2}, a_{5}^{3}\right\}$ & $V(\mathbb{C})$ \\
$R_{72}=R\left\{a_{2}^{2}, a_{7}^{3}\right\}$ & $V(\mathbb{C})$ \\
$R_{74}=R\left\{a_{3}^{2}, a_{2}^{3}\right\}$ & $V(\mathbb{C})$ \\
$R_{76}=R\left\{a_{3}^{2}, a_{8}^{3}\right\}$ & $V(\mathbb{C})$ \\
$R_{78}=R\left\{a_{4}^{2}, a_{4}^{3}\right\}$ & $V(\mathbb{C})$ \\
$R_{80}=R\left\{a_{1}^{3}, a_{4}^{3}\right\}$ & $V(\mathbb{C})$ \\
$R_{82}=R\left\{a_{2}^{3}, a_{5}^{3}\right\}$ & $V(\mathbb{C})$ \\
$R_{84}=R\left\{a_{2}^{3}, a_{9}^{3}\right\}$ & $V(\mathbb{C})$ \\
\hline
\end{tabular}

TABLE 4: Resolving neighbourhood sets of $\mathbb{W} \mathbb{D}(3)$ with minimum cardinality.

\begin{tabular}{lc}
\hline $\begin{array}{l}\text { Resolving } \\
\text { neighbourhood sets }\end{array}$ & Elements \\
\hline$R_{1}=R\left\{a_{1}^{1}, a_{2}^{1}\right\}$ & $V(\mathbb{C})-\left\{a_{2}^{2}, a_{3}^{2}, a_{4}^{2}\right\} \cup\left\{a_{4}^{3}, a_{5}^{3}, a_{6}^{3}, a_{8}^{3}, a_{9}^{3}\right\}$ \\
$R_{2}=R\left\{a_{1}^{1}, a_{2}^{3}\right\}$ & $V(\mathbb{C})-\left\{a_{1}^{2}, a_{2}^{2}, a_{4}^{2}\right\} \cup\left\{a_{1}^{3}, a_{2}^{3}, a_{7}^{3}, a_{8}^{3}, a_{9}^{3}\right\}$ \\
$R_{3}=R\left\{a_{1}^{1}, a_{4}^{2}\right\}$ & $V(\mathbb{C})-\left\{a_{1}^{2}, a_{2}^{2}, a_{3}^{2}\right\} \cup\left\{a_{1}^{3}, a_{2}^{3}, a_{3}^{3}, a_{4}^{3}, a_{5}^{3}\right\}$ \\
$R_{4}=R\left\{a_{2}^{1}, a_{3}^{1}\right\}$ & $V(\mathbb{C})-\left\{a_{2}^{3}, a_{3}^{3}, a_{4}^{3}, a_{5}^{3}, a_{6}^{3}, a_{7}^{3}, a_{8}^{3}, a_{9}^{3}\right\}$ \\
$R_{5}=R\left\{a_{3}^{2}, a_{5}^{3}\right\}$ & $V(\mathbb{C})-\left\{a_{1}^{3}, a_{2}^{3}, a_{3}^{3}, a_{4}^{3}, a_{6}^{3}, a_{7}^{3}, a_{8}^{3}, a_{9}^{3}\right\}$ \\
$R_{6}=R\left\{a_{4}^{2}, a_{9}^{3}\right\}$ & $V(\mathbb{C})-\left\{a_{1}^{3}, a_{2}^{3}, a_{3}^{3}, a_{4}^{3}, a_{5}^{3}, a_{6}^{3}, a_{7}^{3}, a_{8}^{3}\right\}$ \\
\hline
\end{tabular}

30 and $\cup_{L=150}^{240} R_{L}=V(\mathbb{C})$. Table 8 , on the other hand, shows the resolving neighbourhood sets with a minimum cardinality of 10 and $\cup_{t=1}^{3} R_{t}=V(\mathbb{C})-\left\{a_{2}^{3}\right.$, $\left.a_{3}^{3}, a_{4}^{3}, a_{6}^{3}, a_{7}^{3}, a_{8}^{3}\right\} \cup\left\{a_{4}^{4}, a_{10}^{4}, a_{11}^{4}, a_{12}^{4}\right\}$. Suppose that, for $150 \leq L \leq 240, \quad\left|R_{L}\right|=\gamma, \quad$ for $\quad 1 \leq t \leq 3, \quad\left|R_{t}\right|=\lambda$, $\eta=\left|\cup{ }_{L=63}^{84} R_{L}\right|=|V(\mathbb{\mathbb { D }}(3))|=30, \quad$ and $\delta=$ $\left|\cup_{t=1}^{6} R_{t}\right|=|V(\mathbb{D D}(3))|=20$. Now, we define a 
TABLE 5: Resolving neighbourhood sets of $\mathbb{D D}(4)$ that are not equal to each other.

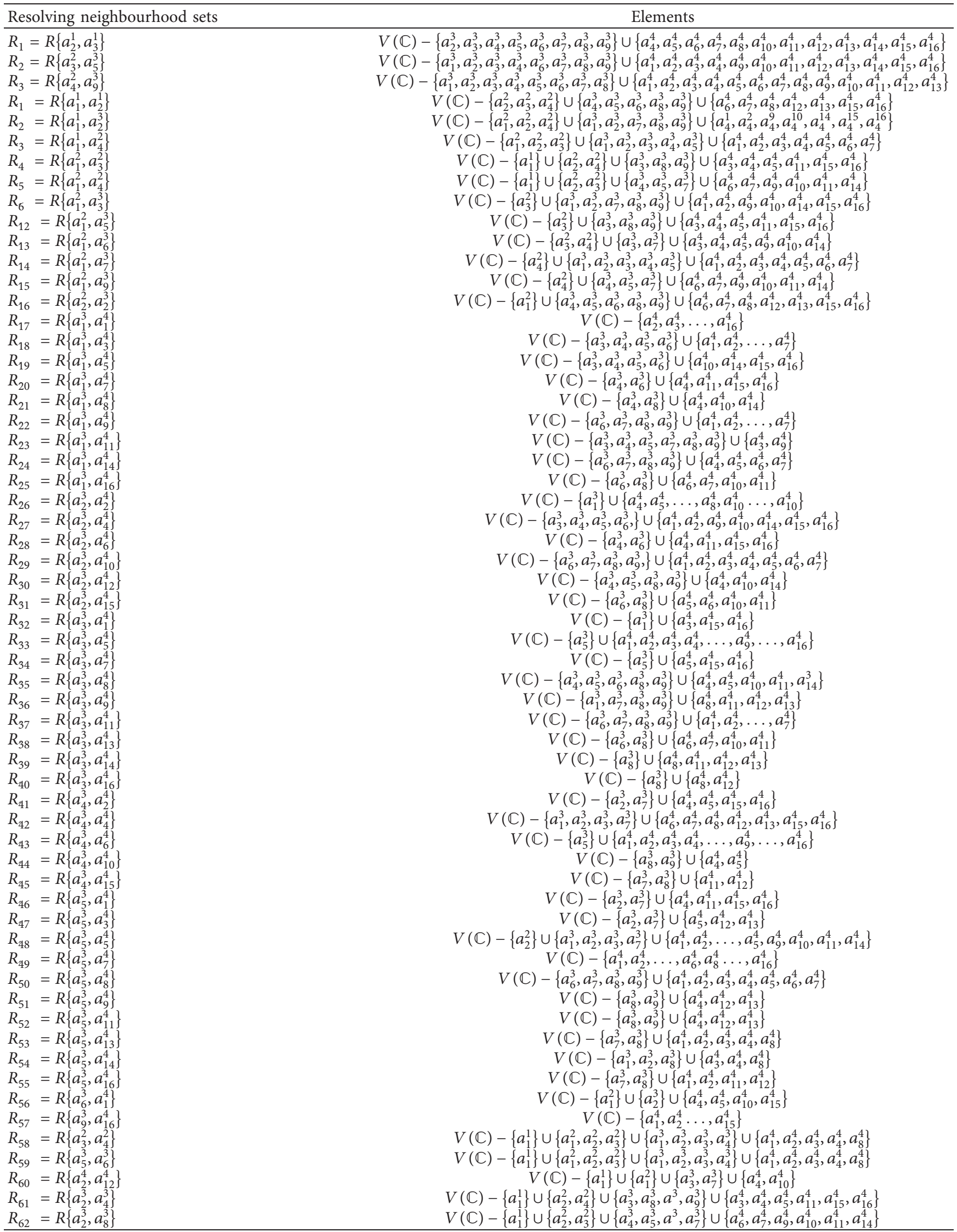


TABle 5: Continued.

\begin{tabular}{|c|c|}
\hline Resolving neighbourhood sets & Elements \\
\hline$R_{63}=R\left\{a_{5}^{3}, a_{7}^{3}\right\}$ & $V(\mathbb{C})-\left\{a_{1}^{1}\right\} \cup\left\{a_{2}^{2}\right\} \cup\left\{a_{3}^{3}, a_{6}^{3}\right\} \cup\left\{a_{3}^{4}, a_{4}^{4}, a_{15}^{4}, a_{16}^{4}\right\}$ \\
\hline$R_{64}=R\left\{a_{3}^{3}, a_{9}^{3}\right\}$ & $V(\mathbb{C})-\left\{a_{1}^{1}\right\} \cup\left\{a_{2}^{2}\right\} \cup\left\{a_{6}^{3}, a_{7}^{3}\right\} \cup\left\{a_{6}^{4}, a_{7}^{4}, a_{9}^{4}, a_{10}^{4}\right\}$ \\
\hline$R_{65}=R\left\{a_{1}^{3}, a_{3}^{3}\right\}$ & $V(\mathbb{C})-\left\{a_{1}^{1}\right\} \cup\left\{a_{1}^{2}, a_{2}^{2}, a_{4}^{2}\right\} \cup\left\{a_{2}^{3}, a_{7}^{3}, a_{8}^{3}\right\} \cup\left\{a_{3}^{4}, a_{10}^{4}, a_{14}^{4}, a_{15}^{4}, a_{16}^{4}\right\}$ \\
\hline$R_{66}=R\left\{a_{1}^{3}, a_{7}^{3}\right\}$ & $V(\mathbb{C})-\left\{a_{1}^{1}\right\} \cup\left\{a_{1}^{2}, a_{2}^{2}, a_{3}^{2}\right\} \cup\left\{a_{2}^{3}, a_{3}^{3}, a_{4}^{3}, a_{5}^{3}\right\} \cup\left\{a_{4}^{4}, a_{5}^{4}, a_{6}^{4}, a_{7}^{4}, a_{9}^{4}\right\}$ \\
\hline$R_{67}=R\left\{a_{1}^{4}, a_{3}^{4}\right\}$ & $V(\mathbb{C})-\left\{a_{1}^{1}\right\} \cup\left\{a_{1}^{2}, a_{2}^{2}, a_{4}^{2}\right\} \cup\left\{a_{1}^{3}, a_{2}^{3}, a_{7}^{3}, a_{8}^{3}, a_{9}^{3}\right\} \cup\left\{a_{2}^{4}, a_{9}^{4}, a_{10}^{4}, a_{14}^{4}, a_{15}^{4}, a_{16}^{4}\right\}$ \\
\hline$R_{68}=R\left\{a_{1}^{4}, a_{7}^{4}\right\}$ & $V(\mathbb{C})-\left\{a_{1}^{1}\right\} \cup\left\{a_{1}^{2}, a_{2}^{2}, a_{3}^{2}\right\} \cup\left\{a_{1}^{3}, a_{2}^{3}, a_{3}^{3}, a_{4}^{3}, a_{5}^{3}\right\} \cup\left\{a_{2}^{4}, a_{3}^{4}, a_{4}^{4}, a_{5}^{4}, a_{6}^{4}, a_{7}^{4}\right\}$ \\
\hline$R_{69}=R\left\{a_{6}^{3}, a_{9}^{3}\right\}$ & $V(\mathbb{C})-\left\{a_{1}^{1}\right\} \cup\left\{a_{1}^{2}, a_{2}^{2}, a_{4}^{2}\right\} \cup\left\{a_{1}^{3}, a_{2}^{3}, a_{7}^{3}, a_{8}^{3}\right\} \cup\left\{a_{1}^{4}, a_{2}^{4}, a_{9}^{4}, a_{10}^{4}, a_{13}^{4}\right\}$ \\
\hline$R_{70}=R\left\{a_{5}^{3}, a_{9}^{3}\right\}$ & $V(\mathbb{C})-\left\{a_{1}^{1}\right\} \cup\left\{a_{1}^{2}, a_{2}^{2}\right\} \cup\left\{a_{1}^{3}, a_{2}^{3}, a_{6}^{3}\right\} \cup\left\{a_{1}^{4}, a_{2}^{4}, a_{11}^{4}, a_{12}^{4}\right\}$ \\
\hline$R_{71}=R\left\{a_{5}^{4}, a_{14}^{4}\right\}$ & $V(\mathbb{C})-\left\{a_{1}^{1}\right\} \cup\left\{a_{1}^{2}, a_{2}^{2}\right\} \cup\left\{a_{1}^{3}, a_{2}^{3}, a_{6}^{3}\right\} \cup\left\{a_{1}^{4}, a_{2}^{4}, a_{11}^{4}, a_{12}^{4}\right\}$ \\
\hline$R_{72}=R\left\{a_{7}^{4}, a_{16}^{4}\right\}$ & $V(\mathbb{C})-\left\{a_{1}^{1}\right\} \cup\left\{a_{1}^{2}, a_{2}^{2}\right\} \cup\left\{a_{1}^{3}, a_{2}^{3}, a_{6}^{3}\right\} \cup\left\{a_{1}^{4}, a_{2}^{4}, a_{11}^{4}, a_{12}^{4}\right\}$ \\
\hline$R_{73}=R\left\{a_{1}^{1}, a_{1}^{4}\right\}$ & $V(\mathbb{C})-\left\{a_{2}^{3}, a_{3}^{3}, a_{7}^{3}\right\} \cup\left\{a_{6}^{4}, a_{7}^{4}, a_{8}^{4}, a_{12}^{4}, a_{13}^{4}, a_{15}^{4}, a_{16}^{4}\right\}$ \\
\hline$R_{74}=R\left\{a_{1}^{1}, a_{7}^{4}\right\}$ & $V(\mathbb{C})-\left\{a_{3}^{3}, a_{4}^{3}, a_{6}^{3}\right\} \cup\left\{a_{1}^{4}, a_{2}^{4}, a_{9}^{4}, a_{10}^{4}, a_{14}^{4}, a_{15}^{4}, a_{16}^{4}\right\}$ \\
\hline$R_{75}=R\left\{a_{1}^{1}, a_{9}^{4}\right\}$ & $V(\mathbb{C})-\left\{a_{6}^{3}, a_{7}^{3}, a_{8}^{3}\right\} \cup\left\{a_{1}^{4}, a_{2}^{4}, a_{3}^{4}, a_{4}^{4}, a_{5}^{4}, a_{6}^{4}, a_{7}^{4}\right\}$ \\
\hline$R_{76}=R\left\{a_{2}^{3}, a_{9}^{4}\right\}$ & $V(\mathbb{C})-\left\{a_{6}^{3}, a_{7}^{3}, a_{8}^{3}\right\} \cup\left\{a_{1}^{4}, a_{2}^{4}, a_{3}^{4}, a_{4}^{4}, a_{5}^{4}, a_{6}^{4}, a_{7}^{4}\right\}$ \\
\hline$R_{77}=R\left\{a_{9}^{3}, a_{14}^{4}\right\}$ & $V(\mathbb{C})-\left\{a_{1}^{2}\right\} \cup\left\{a_{1}^{3}, a_{2}^{3}, a_{3}^{3}, a_{7}^{3}\right\} \cup\left\{a_{1}^{4}, a_{2}^{4}, \ldots, a_{5}^{4}, a_{9}^{4}, a_{10}^{4}, a_{11}^{4}, a_{14}^{4}\right\}$ \\
\hline$R_{78}=R\left\{a_{2}^{3}, a_{2}^{4}\right\}$ & $V(\mathbb{C})-\left\{a_{1}^{3}\right\} \cup\left\{a_{4}^{4}, a_{5}^{4}, \ldots, a_{8}^{4}, a_{10}^{4}, \ldots, a_{16}^{4}\right\}$ \\
\hline
\end{tabular}

mapping $\quad \kappa: V(\mathbb{\square} \mathbb{D}(3)) \longrightarrow[0,1]$ such that $\kappa(a)=(1 / 30)$ for all $a \in \cup_{L=63}^{84} R_{L}$, and assigning the value of $(1 / 30)$ to all the elements of $\cup_{L=63}^{84} R_{L}$ and summing up all the labels, we get $|\kappa|$ $=\sum_{a \in \cup_{L=63}^{84} R_{L}} \kappa(a)=(30 / 30)=1 ;$ thus, $\operatorname{dim}_{\text {frac }}(\mathbb{C})=$ $|\kappa|=1$, as all the $R_{L}$ for $150 \leq L \leq 240$ are all having $V(\mathbb{D}(3))$ in common. Similarly, we define another mapping $\tau: V(\mathbb{W}(3)) \longrightarrow[0,1]$ such that $\tau=(1 / \lambda)=$ $(1 / 10)$ for all $a \in \cup_{t=1}^{3} R_{t}$, and giving labels to the elements of $\cup_{t=1}^{3} R_{t}$ and latter on summing them up, we get $|\tau|=\sum_{a \in \cup_{t=1}^{3} R_{t}} \tau(a)=(20 / 10) \leq 2$; hence, $\operatorname{dim}_{\text {frac }}(\mathbb{C})<2$ as all the $R_{t}$ for $1 \leq t \leq 6$ are pairwise overlapping. Therefore, we arrive at the following conclusion:

$$
1<\operatorname{dim}_{\text {frac }}(\mathbb{W}(3)) \leq 2 \text {. }
$$

Case 2: when $n \geq 6$.

The required minimum resolving neighbourhood sets are $R\left\{a_{1}^{\alpha}, a_{1}^{\beta}\right\}, R\left\{a_{\gamma}^{\alpha}, a_{\eta}^{\beta}\right\}$, and $R\left\{a_{\alpha^{2}}^{\alpha}, a_{\beta^{2}}^{\beta}\right\}$, where $\alpha=(n / 2), \beta+1, \gamma=2 \alpha-1$, and $\eta=2 \beta-1$. As it is evident from Lemma $1, \quad\left|R_{t}\right|=\left|R\left\{a_{1}^{\alpha}, a_{1}^{\beta}\right\}\right|$ $=\left|R\left\{a_{\gamma}^{\alpha}, a_{\eta}^{\beta}\right\}\right|=\mid R\left\{a_{\alpha^{2}}^{\alpha}, a_{\beta^{2}}^{\beta} \mid=\quad\left(n\left(n^{2}+3 n+2\right)\right.\right.$ /12) $\leq \|$ acute $R\{a, b\} \mid$ for all $a, b \in V(\mathbb{C})$ and $\cup_{t=1}^{3} R_{t}=\cup_{j=1}^{\alpha} S_{j^{2}}^{j} \cup \cup_{j=\alpha+1}^{n}\left(S_{(j-\alpha)^{2}}^{j, t} \cup S_{(j-\alpha)^{2}}^{j, b r} \cup S_{(j-\alpha)^{2}}^{j, b l}\right)$. Moreover, $\left|R\{u, v\} \cap \cup_{t=1}^{3} R_{t}\right| \geq\left|R_{t}\right|$ for all $u, v \in V(\mathbb{C})$. Also, by Lemma 3 (d), the resolving neighbourhood sets with maximum cardinality of $|V(\mathbb{C})|=\left(n\left(2 n^{2}+\right.\right.$ $3 n+1) / 6)$ are $R_{1}=R\left\{a_{\alpha}^{\alpha}, a_{\beta}^{\beta}\right\}$ and $R_{2}=R\left\{a_{\alpha}^{\alpha}, a_{\alpha+\gamma}^{\alpha+\eta}\right\}$ with, $\cup_{L=1}^{2} R_{L}=V(\mathbb{C})$. Let $\left|R_{t}\right|=\lambda, \quad\left|R_{L}\right|=\gamma$, $\left|\cup_{L=1}^{2} R_{L}\right|=\eta$, and $\left|\cup_{t=1}^{3} R_{t}\right|=\delta$; to find the minimum value of $\operatorname{dim}_{\text {frac }}(\mathbb{C})$, we define a mapping $\kappa: V(\mathbb{C}) \longrightarrow[0,1]$ such that

$$
k(a)= \begin{cases}\frac{1}{k}, & \text { for } a \in \bigcup_{L=1}^{3} R_{t}, \\ 0, & \text { for } a \in V(\mathbb{C})-\bigcup_{t=1}^{3} R_{L} .\end{cases}
$$

TABLE 6: Resolving neighbourhood sets of $\mathbb{U D}(4)$ that are equal due to symmetry.

\begin{tabular}{llcl}
\hline $\begin{array}{l}\text { Resolving } \\
\text { neighbourhood sets }\end{array}$ & Equality & $\begin{array}{c}\text { Resolving } \\
\text { neighbourhood sets }\end{array}$ & Equality \\
\hline$R_{79}=R\left\{a_{1}^{3}, a_{13}^{4}\right\}$ & $R\left\{a_{1}^{3}, a_{8}^{4}\right\}$ & $R_{80}=R\left\{a_{3}^{3}, a_{6}^{3}\right\}$ & $R\left\{a_{1}^{2}, a_{4}^{2}\right\}$ \\
$R_{81}=R\left\{a_{5}^{4}, a_{8}^{4}\right\}$ & $R\left\{a_{1}^{2}, a_{4}^{2}\right\}$ & $R_{82}=R\left\{a_{7}^{5}, a_{10}^{5}\right\}$ & $R\left\{a_{1}^{2}, a_{4}^{2}\right\}$ \\
$R_{83}=R\left\{a_{6}^{3}, a_{7}^{3}\right\}$ & $R\left\{a_{1}^{2}, a_{3}^{2}\right\}$ & $R_{84}=R\left\{a_{13}^{4}, a_{14}^{4}\right\}$ & $R\left\{a_{1}^{2}, a_{3}^{2}\right\}$ \\
$R_{85}=R\left\{a_{2}^{2}, a_{4}^{3}\right\}$ & $R\left\{a_{1}^{2}, a_{3}^{3}\right\}$ & $R_{86}=R\left\{a_{2}^{2}, a_{8}^{3}\right\}$ & $R\left\{a_{1}^{2}, a_{7}^{3}\right\}$ \\
$R_{87}=R\left\{a_{3}^{2}, a_{3}^{3}\right\}$ & $R\left\{a_{2}^{2}, a_{2}^{3}\right\}$ & $R_{88}=R\left\{a_{4}^{2}, a_{7}^{3}\right\}$ & $R\left\{a_{2}^{2}, a_{2}^{3}\right\}$ \\
$R_{89}=R\left\{a_{3}^{2}, a_{6}^{3}\right\}$ & $R\left\{a_{1}^{2}, a_{7}^{3}\right\}$ & $R_{90}=R\left\{a_{4}^{2}, a_{6}^{3}\right\}$ & $R\left\{a_{1}^{2}, a_{3}^{3}\right\}$ \\
$R_{91}=R\left\{a_{5}^{4}, a_{11}^{4}\right\}$ & $R\left\{a_{5}^{3}, a_{6}^{3}\right\}$ & $R_{92}=R\left\{a_{5}^{4}, a_{11}^{4}\right\}$ & $R\left\{a_{5}^{3}, a_{6}^{3}\right\}$ \\
$R_{93}=R\left\{a_{7}^{4}, a_{13}^{4}\right\}$ & $R\left\{a_{5}^{3}, a_{6}^{3}\right\}$ & $R_{94}=R\left\{a_{11}^{4}, a_{14}^{4}\right\}$ & $R\left\{a_{6}^{3}, a_{9}^{3}\right\}$ \\
$R_{95}=R\left\{a_{12}^{4}, a_{15}^{4}\right\}$ & $R\left\{a_{6}^{3}, a_{9}^{3}\right\}$ & $R_{96}=R\left\{a_{8}^{4}, a_{16}^{4}\right\}$ & $R\left\{a_{6}^{3}, a_{9}^{3}\right\}$ \\
$R_{97}=R\left\{a_{5}^{4}, a_{14}^{4}\right\}$ & $R\left\{a_{5}^{3}, a_{9}^{3}\right\}$ & $R_{98}=R\left\{a_{5}^{4}, a_{14}^{4}\right\}$ & $R\left\{a_{5}^{3}, a_{9}^{3}\right\}$ \\
$R_{99}=R\left\{a_{7}^{4}, a_{13}^{4}\right\}$ & $R\left\{a_{5}^{3}, a_{6}^{3}\right\}$ & $R_{100}=R\left\{a_{8}^{4}, a_{16}^{4}\right\}$ & $R\left\{a_{6}^{3}, a_{9}^{3}\right\}$ \\
$R_{\mathrm{r} 01}=R\left\{a_{2}^{4}, a_{10}^{4}\right\}$ & $R\left\{a_{1}^{3}, a_{7}^{3}\right\}$ & $R_{102}=R\left\{a_{2}^{4}, a_{4}^{4}\right\}$ & $R\left\{a_{1}^{3}, a_{3}^{3}\right\}$ \\
$R_{\mathrm{r} 03}=R\left\{a_{3}^{3}, a_{3}^{4}\right\}$ & $R\left\{a_{2}^{3}, a_{2}^{4}\right\}$ & $R_{\mathrm{r} 04}=R\left\{a_{3}^{4}, a_{11}^{4}\right\}$ & $R\left\{a_{1}^{3}, a_{7}^{3}\right\}$ \\
$R_{\mathrm{r} 05}=R\left\{a_{9}^{4}, a_{11}^{4}\right\}$ & $R\left\{a_{1}^{3}, a_{3}^{3}\right\}$ & $R_{106}=R\left\{a_{6}^{3}, a_{11}^{4}\right\}$ & $R\left\{a_{9}^{3}, a_{14}^{4}\right\}$ \\
$R_{107}=R\left\{a_{5}^{3}, a_{5}^{4}\right\}$ & $R\left\{a_{9}^{3}, a_{14}^{4}\right\}$ & $R_{108}=R\left\{a_{3}^{3}, a_{3}^{4}\right\}$ & $R\left\{a_{2}^{3}, a_{2}^{4}\right\}$ \\
\hline
\end{tabular}

where $\gamma=\eta=\left(n\left(2 n^{2}+3 n+1\right) / 6\right)$. Assigning the labels to the elements of $\bigcup \cup_{L=1}^{2} R_{L}$ and summing them up, we get $|\kappa|=\sum_{a \in \cup_{L=1}^{2} R_{L}}(1 / \gamma)=1$.

Similarly, for the maximum value of $\operatorname{dim}_{\text {frac }}(\mathbb{C})$, we define another mapping $\tau: V(\mathbb{C}) \longrightarrow[0,1]$ such that

$$
\tau(a)= \begin{cases}\frac{1}{\lambda}, & \text { for } a \in \cup_{t=1}^{3} R_{t}, \\ 0, & \text { for } a \in V(\mathbb{C})-\bigcup_{t=1}^{3} R_{t} .\end{cases}
$$

It can be seen that $\tau$ is a resolving function for $\mathbb{C}$ with $n \geq 3$ because $\tau(R\{u, v\}) \geq 1 \forall u, v \in V(\mathbb{N})$. On the contrary, we assume that there is another resolving function $\rho$ such that $\rho(u) \leq \tau(u)$, for at least one $u \in V(\mathbb{C}) \rho(u) \neq \tau(u)$. As a consequence, $\rho(R\{u, v\})<1$, where $R\{u, v\}$ is a resolving neighbourhood of $\mathbb{C}$ with minimum cardinality $\kappa$. It shows 
TABLE 7: Resolving neighbourhood sets of $\mathbb{U D}(4)$ that are equal to $V(\mathbb{D D}(4))$.

\begin{tabular}{|c|c|c|c|}
\hline Resolving neighbourhood sets & Elements & Resolving neighbourhood sets & Elements \\
\hline$R_{r_{50}}=R\left\{a_{1}^{1}, a_{2}^{3}\right\}$ & $V(\mathbb{C})$ & $R_{\mathrm{r} 51}=R\left\{a_{1}^{1}, a_{4}^{3}\right\}$ & $V(\mathbb{C})$ \\
\hline$R_{\mathrm{r} 52}=R\left\{a_{1}^{1}, a_{8}^{3}\right\}$ & $V(\mathbb{C})$ & $R_{\mathrm{r} 53}=R\left\{a_{1}^{1}, a_{2}^{4}\right\}$ & $V(\mathbb{C})$ \\
\hline$R_{154}=R\left\{a_{1}^{1}, a_{4}^{4}\right\}$ & $V(\mathbb{C})$ & $R_{155}=R\left\{a_{1}^{1}, a_{6}^{4}\right\}$ & $V(\mathbb{C})$ \\
\hline$R_{\mathrm{r} 56}=R\left\{a_{1}^{1}, a_{10}^{4}\right\}$ & $V(\mathbb{C})$ & $R_{157}=R\left\{a_{1}^{1}, a_{12}^{4}\right\}$ & $V(\mathbb{C})$ \\
\hline$R_{158}=R\left\{a_{1}^{1}, a_{15}^{4}\right\}$ & $V(\mathbb{C})$ & $R_{\mathrm{r} 59}=R\left\{a_{1}^{2}, a_{2}^{3}\right\}$ & $V(\mathbb{C})$ \\
\hline$R_{\mathrm{r} 60}=R\left\{a_{1}^{2}, a_{4}^{3}\right\}$ & $V(\mathbb{C})$ & $R_{161}=R\left\{a_{1}^{2}, a_{8}^{3}\right\}$ & $V(\mathbb{C})$ \\
\hline$R_{\mathrm{r} 62}=R\left\{a_{2}^{2}, a_{1}^{3}\right\}$ & $V(\mathbb{C})$ & $R_{\mathrm{r} 63}=R\left\{a_{2}^{2}, a_{3}^{3}\right\}$ & $V(\mathbb{C})$ \\
\hline$R_{\mathrm{r} 64}=R\left\{a_{2}^{2}, a_{5}^{3}\right\}$ & $V(\mathbb{C})$ & $R_{\mathrm{Y} 65}=R\left\{a_{2}^{2}, a_{6}^{3}\right\}$ & $V(\mathbb{C})$ \\
\hline$R_{\text {r66 }}=R\left\{a_{2}^{2}, a_{7}^{3}\right\}$ & $V(\mathbb{C})$ & $R_{\mathrm{r} 67}=R\left\{a_{2}^{2}, a_{9}^{3}\right\}$ & $V(\mathbb{C})$ \\
\hline$R_{\mathrm{r} 68}=R\left\{a_{3}^{2}, a_{2}^{3}\right\}$ & $V(\mathbb{C})$ & $R_{\mathrm{r} 69}=R\left\{a_{3}^{2}, a_{4}^{3}\right\}$ & $V(\mathbb{C})$ \\
\hline$R_{\mathrm{r} 70}=R\left\{a_{3}^{2}, a_{8}^{3}\right\}$ & $V(\mathbb{C})$ & $R_{\mathrm{r} 71}=R\left\{a_{4}^{2}, a_{2}^{3}\right\}$ & $V(\mathbb{C})$ \\
\hline$R_{172}=R\left\{a_{4}^{2}, a_{4}^{3}\right\}$ & $V(\mathbb{C})$ & $R_{\mathrm{r} 73}=R\left\{a_{4}^{2}, a_{8}^{3}\right\}$ & $V(\mathbb{C})$ \\
\hline$R_{\mathrm{r} 74}=R\left\{a_{1}^{3}, a_{4}^{3}\right\}$ & $V(\mathbb{C})$ & $R_{\mathrm{r} 75}=R\left\{a_{1}^{3}, a_{8}^{3}\right\}$ & $V(\mathbb{C})$ \\
\hline$R_{\mathrm{r} 76}=R\left\{a_{2}^{\frac{1}{3}}, a_{5}^{3}\right\}$ & $V(\mathbb{C})$ & $R_{\mathrm{r} 77}=R\left\{a_{2}^{\frac{1}{3}}, a_{6}^{3}\right\}$ & $V(\mathbb{C})$ \\
\hline$R_{178}=R\left\{a_{2}^{3}, a_{9}^{3}\right\}$ & $V(\mathbb{C})$ & $R_{\mathrm{r} 79}=R\left\{a_{1}^{4}, a_{4}^{4}\right\}$ & $V(\mathbb{C})$ \\
\hline$R_{180}=R\left\{a_{1}^{4}, a_{10}^{4}\right\}$ & $V(\mathbb{C})$ & $R_{181}=R\left\{a_{1}^{4}, a_{6}^{4}\right\}$ & $V(\mathbb{C})$ \\
\hline$R_{182}=R\left\{a_{1}^{4}, a_{12}^{4}\right\}$ & $V(\mathbb{C})$ & $R_{\mathrm{r} 83}=R\left\{a_{1}^{4}, a_{15}^{4}\right\}$ & $V(\mathbb{C})$ \\
\hline$R_{\mathrm{r} 84}=R\left\{a_{2}^{4}, a_{5}^{4}\right\}$ & $V(\mathbb{C})$ & $R_{185}=R\left\{a_{2}^{4}, a_{11}^{4}\right\}$ & $V(\mathbb{C})$ \\
\hline$R_{186}=R\left\{a_{2}^{4}, a_{14}^{4}\right\}$ & $V(\mathbb{C})$ & $R_{187}=R\left\{a_{2}^{4}, a_{7}^{4}\right\}$ & $V(\mathbb{C})$ \\
\hline$R_{\mathrm{r} 88}=R\left\{a_{2}^{4}, a_{8}^{4}\right\}$ & $V(\mathbb{C})$ & $R_{189}=R\left\{a_{2}^{4}, a_{13}^{4}\right\}$ & $V(\mathbb{C})$ \\
\hline$R_{\mathrm{r90}}=R\left\{a_{2}^{4}, a_{16}^{4}\right\}$ & $V(\mathbb{C})$ & $R_{191}=R\left\{a_{3}^{4}, a_{6}^{4}\right\}$ & $V(\mathbb{C})$ \\
\hline$R_{192}=R\left\{a_{3}^{4}, a_{12}^{4}\right\}$ & $V(\mathbb{C})$ & $R_{193}=R\left\{a_{3}^{4}, a_{15}^{4}\right\}$ & $V(\mathbb{C})$ \\
\hline$R_{\mathrm{r} 94}=R\left\{a_{4}^{4}, a_{7}^{4}\right\}$ & $V(\mathbb{C})$ & $R_{195}=R\left\{a_{4}^{4}, a_{8}^{4}\right\}$ & $V(\mathbb{C})$ \\
\hline$R_{\mathrm{r96}}=R\left\{a_{4}^{4}, a_{13}^{4}\right\}$ & $V(\mathbb{C})$ & $R_{197}=R\left\{a_{4}^{4}, a_{16}^{4}\right\}$ & $V(\mathbb{C})$ \\
\hline$R_{198}=R\left\{a_{1}^{1}, a_{2}^{4}\right\}$ & $V(\mathbb{C})$ & $R_{199}=R\left\{a_{1}^{1}, a_{4}^{4}\right\}$ & $V(\mathbb{C})$ \\
\hline$R_{200}=R\left\{a_{1}^{1}, a_{6}^{4}\right\}$ & $V(\mathbb{C})$ & $R_{201}=R\left\{a_{1}^{1}, a_{12}^{4}\right\}$ & $V(\mathbb{C})$ \\
\hline$R_{202}=R\left\{a_{1}^{1}, a_{15}^{4}\right\}$ & $V(\mathbb{C})$ & $R_{203}=R\left\{a_{1}^{2}, a_{2}^{4}\right\}$ & $V(\mathbb{C})$ \\
\hline$R_{204}=R\left\{a_{1}^{2}, a_{4}^{4}\right\}$ & $V(\mathbb{C})$ & $R_{205}=R\left\{a_{1}^{2}, a_{6}^{4}\right\}$ & $V(\mathbb{C})$ \\
\hline$R_{206}=R\left\{a_{1}^{2}, a_{12}^{4}\right\}$ & $V(\mathbb{C})$ & $R_{207}=R\left\{a_{1}^{2}, a_{4}^{4}\right\}$ & $V(\mathbb{C})$ \\
\hline$R_{208}=R\left\{a_{1}^{2}, a_{6}^{4}\right\}$ & $V(\mathbb{C})$ & $R_{209}=R\left\{a_{1}^{2}, a_{12}^{4}\right\}$ & $V(\mathbb{C})$ \\
\hline$R_{210}=R\left\{a_{2}^{2}, a_{1}^{4}\right\}$ & $V(\mathbb{C})$ & $R_{211}=R\left\{a_{2}^{2}, a_{3}^{4}\right\}$ & $V(\mathbb{C})$ \\
\hline$R_{212}=R\left\{a_{2}^{2}, a_{5}^{4}\right\}$ & $V(\mathbb{C})$ & $R_{213}=R\left\{a_{2}^{2}, a_{7}^{4}\right\}$ & $V(\mathbb{C})$ \\
\hline$R_{214}=R\left\{a_{2}^{2}, a_{8}^{4}\right\}$ & $V(\mathbb{C})$ & $R_{215}=R\left\{a_{2}^{2}, a_{11}^{4}\right\}$ & $V(\mathbb{C})$ \\
\hline$R_{216}=R\left\{a_{2}^{2}, a_{13}^{4}\right\}$ & $V(\mathbb{C})$ & $R_{217}=R\left\{a_{2}^{2}, a_{16}^{4}\right\}$ & $V(\mathbb{C})$ \\
\hline$R_{218}=R\left\{a_{3}^{2}, a_{2}^{4}\right\}$ & $V(\mathbb{C})$ & $R_{219}=R\left\{a_{3}^{2}, a_{4}^{4}\right\}$ & $V(\mathbb{C})$ \\
\hline$R_{220}=R\left\{a_{3}^{2}, a_{6}^{4}\right\}$ & $V(\mathbb{C})$ & $R_{221}=R\left\{a_{3}^{2}, a_{10}^{4}\right\}$ & $V(\mathbb{C})$ \\
\hline$R_{222}=R\left\{a_{3}^{2}, a_{12}^{4}\right\}$ & $V(\mathbb{C})$ & $R_{223}=R\left\{a_{3}^{2}, a_{15}^{4}\right\}$ & $V(\mathbb{C})$ \\
\hline$R_{224}=R\left\{a_{4}^{2}, a_{2}^{4}\right\}$ & $V(\mathbb{C})$ & $R_{225}=R\left\{a_{4}^{2}, a_{4}^{4}\right\}$ & $V(\mathbb{C})$ \\
\hline$R_{226}=R\left\{a_{4}^{2}, a_{6}^{4}\right\}$ & $V(\mathbb{C})$ & $R_{227}=R\left\{a_{4}^{2}, a_{10}^{4}\right\}$ & $V(\mathbb{C})$ \\
\hline$R_{228}=R\left\{a_{4}^{2}, a_{12}^{4}\right\}$ & $V(\mathbb{C})$ & $R_{229}=R\left\{a_{4}^{2}, a_{15}^{4}\right\}$ & $V(\mathbb{C})$ \\
\hline$R_{230}=R\left\{a_{1}^{3}, a_{2}^{4}\right\}$ & $V(\mathbb{C})$ & $R_{231}=R\left\{a_{1}^{3}, a_{4}^{4}\right\}$ & $V(\mathbb{C})$ \\
\hline$R_{232}=R\left\{a_{1}^{3}, a_{6}^{4}\right\}$ & $V(\mathbb{C})$ & $R_{233}=R\left\{a_{1}^{3}, a_{12}^{4}\right\}$ & $V(\mathbb{C})$ \\
\hline$R_{234}=R\left\{a_{1}^{3}, a_{15}^{4}\right\}$ & $V(\mathbb{C})$ & $R_{235}=R\left\{a_{2}^{3}, a_{1}^{4}\right\}$ & $V(\mathbb{C})$ \\
\hline$R_{236}=R\left\{a_{2}^{3}, a_{3}^{4}\right\}$ & $V(\mathbb{C})$ & $R_{237}=R\left\{a_{2}^{3}, a_{5}^{4}\right\}$ & $V(\mathbb{C})$ \\
\hline$R_{238}=R\left\{a_{2}^{3}, a_{7}^{4}\right\}$ & $V(\mathbb{C})$ & $R_{239}=R\left\{a_{2}^{3}, a_{8}^{4}\right\}$ & $V(\mathbb{C})$ \\
\hline$R_{240}=R\left\{a_{2}^{3}, a_{11}^{4}\right\}$ & $V(\mathbb{C})$ & & \\
\hline
\end{tabular}

TABLE 8: Resolving neighbourhood sets of $\mathbb{D D}(4)$ that are not equal to each other.

\begin{tabular}{lc}
\hline Resolving neighbourhood sets & Elements \\
\hline$R_{1}=R\left\{a_{2}^{1}, a_{3}^{1}\right\}$ & $V(\mathbb{C})-\left\{a_{2}^{3}, a_{3}^{3}, a_{4}^{3}, a_{5}^{3}, a_{6}^{3}, a_{7}^{3}, a_{8}^{3}, a_{9}^{3}\right\} \cup\left\{a_{4}^{4}, a_{5}^{4}, a_{6}^{4}, a_{7}^{4}, a_{8}^{4}, a_{10}^{4}, a_{11}^{4}, a_{12}^{4}, a_{13}^{4}, a_{14}^{4}, a_{15}^{4}, a_{16}^{4}\right\}$ \\
$R_{2}=R\left\{a_{3}^{2}, a_{5}^{3}\right\}$ & $V(\mathbb{C})-\left\{a_{1}^{3}, a_{2}^{3}, a_{3}^{3}, a_{4}^{3}, a_{6}^{3}, a_{7}^{3}, a_{8}^{3}, a_{9}^{3}\right\} \cup\left\{a_{1}^{4}, a_{2}^{4}, a_{3}^{4}, a_{4}^{4}, a_{9}^{4}, a_{10}^{4}, a_{11}^{4}, a_{12}^{4}, a_{13}^{4}, a_{14}^{4}, a_{15}^{4}, a_{16}^{4}\right\}$ \\
$R_{3}=R\left\{a_{4}^{2}, a_{9}^{3}\right\}$ & $V(\mathbb{C})-\left\{a_{1}^{3}, a_{2}^{3}, a_{3}^{3}, a_{4}^{3}, a_{5}^{3}, a_{6}^{3}, a_{7}^{3}, a_{8}^{3}\right\} \cup\left\{a_{1}^{4}, a_{2}^{4}, a_{3}^{4}, a_{4}^{4}, a_{5}^{4}, a_{6}^{4}, a_{7}^{4}, a_{8}^{4}, a_{9}^{4}, a_{10}^{4}, a_{11}^{4}, a_{12}^{4}, a_{13}^{4}\right\}$ \\
\hline
\end{tabular}


that $\rho$ is not a resolving function which is a contradiction. Therefore, $\tau$ is a minimal resolving function that attains minimum $|\tau|$ for $\mathbb{C}$. Since $1 \leq t \leq 3 R_{t}$ has a nonempty intersection, thus there exists another minimal resolving function $\bar{\tau}$ of $\mathbb{C}$ such that $|\bar{\tau}| \leq|\tau|$. Thus, assigning $(1 / \lambda)$ to the vertices of $\mathbb{C}$ in $\cup_{t=1}^{3} R_{t}$ and calculating the summation of all the weights, we get $\operatorname{dim}_{\text {frac }}(\mathbb{C})=\sum_{t=1}^{\delta}(1 / \lambda) \leq\left(12 n\left(n^{2}+\right.\right.$ $\left.3 n+2) / 6 n\left(n^{2}+3 n+2\right)\right)=2$. Therefore, we arrive at the following result:

$$
1<\operatorname{dim}_{\text {frac }}(\mathbb{C}) \leq 2
$$

\section{Conclusions}

We conclude our discussion by the following remarks:

(i) In this article, we have made a characterization of graphs having the FMD as unity

(ii) It is computed that the FMD of the path is 1 that strengthens the result proved in [13]

(iii) We have calculated the extremal values of FMD of $\mathbb{U D}(n)$ as (i) for $n \equiv 0(\bmod 2), 1<\operatorname{dim}_{\text {frac }}(\mathbb{C}) \leq 2$ and (ii) for $n \equiv 1(\bmod 2)$, $1<\operatorname{dim}_{\text {frac }}(\mathbb{C}) \leq 2\left(n^{3}+6 n^{2}+11 n-30 / n^{3}+3 n^{2}+\right.$ $5 n+3)$

(iv) Now, we close our discussion with the open problem that investigates the families of graphs other than $P_{n}$ having FMD as unity

\section{Data Availability}

All the data are included within this article. However, the reader may contact the corresponding author for more details of the data.

\section{Conflicts of Interest}

The authors have no conflicts of interest.

\section{References}

[1] P. J. Slater, "Leaves of trees," Congruent number, vol. 14, no. 1, pp. 549-559, 1975.

[2] P. J. Slater, "Domination and location in acyclic graphs," Networks, vol. 17, no. 1, pp. 55-64, 1987.

[3] F. Harary and R. A. Melter, "On the metric dimension of a graph,” Ars Combinatoria, vol. 2, no. 1, pp. 191-195, 1976.

[4] I. Javaid, M. T. Rahim, and K. Ali, "Families of regular graphs with constant metric dimension," Utilitas Mathematica, vol. 75, no. 1, pp. 21-33, 2008.

[5] I. Tomescu and I. Javaid, "On the metric dimension of the Jahangir graph," Bulletin mathématique de la Société des Sciences, vol. 50, no. 98, pp. 371-376, 2007.

[6] M. Imran, A. Q. Baig, M. K. Shafiq, and I. Tomescu, "On metric dimension of generalized Petersen graphs $P(n, 3)$," ARS Combinatoria, vol. 117, no. 1, pp. 113-130, 2014.

[7] M. Fehr, S. Gosselin, and O. R. Oellermann, "The metric dimension of Cayley digraphs," Discrete Mathematics, vol. 306, no. 1, pp. 31-41, 2006.
[8] R. A. Melter and I. Tomescu, "Metric bases in digital geometry," Computer Vision, Graphics, and Image Processing, vol. 25, no. 1, pp. 113-121, 2014.

[9] S. Khuller, B. Raghavachari, and A. Rosenfield, "Landmarks in graphs," Discrete Applied Mathematics, vol. 70, no. 1, pp. 217-229, 1996.

[10] P. S. Buczkowski, G. Chartrand, C. Poisson, and P. Zhang, "On k- dimensional graphs and their bases," Periodica Mathematica Hungarica, vol. 46, no. 1, pp. 9-15, 2003.

[11] G. Chartrand, L. Eroh, M. A. Johnson, and O. R. Oellermann, "Resolvability in graphs and the metric dimension of a graph," Discrete Applied Mathematics, vol. 105, no. 1-3, pp. 99-113, 2000.

[12] J. Currie and O. R. Oellermann, "The metric dimension and metric independence of a graph," Journal of Combinatorial Mathematics and Combinatorial, vol. 39, no. 1, pp. 157-167, 2001.

[13] S. Arumugam and V. Mathew, "The fractional metric dimension of graphs," Discrete Mathematics, vol. 312, no. 9, pp. 1584-1590, 2012.

[14] S. Arumugam, V. Mathew, and J. Shen, "On fractional metric dimension of graphs Discrete Mathematics," Algorithms and Applications, vol. 5, no. 4, pp. 1-8, 2013.

[15] M. Feng, B. Lv, and K. Wang, "On the fractional metric dimension of graphs," Discrete Applied Mathematics, vol. 170, no. 19, pp. 55-63, 2014.

[16] M. Feng and K. Wang, "On the metric dimension and fractional metric dimension of the hierarchical product of graphs," Applicable Analysis and Discrete Mathematics, vol. 7, no. 1, pp. 302-313, 2013.

[17] S. W. Saputro, A. Semanicova Fenovcikova, M. Baca, and M. Lascsakova, "On fractional metric dimension of comb product graphs," Statistics, Optimization \& Information Computing, vol. 6, no. 1, pp. 150-158, 2018.

[18] M. Feng and K. Wang, "On the fractional metric dimension of corona product graphs and lexicographic product graph $\sigma$," https://arxiv.org/abs/1206.1906.

[19] B. Jia, A. Kashif, T. Rasheed, and M. Javaid, Fractional metri dimension of generalized Jahangir graph," Mathematics, vol. 4, no. 1, pp. 371-376, 2019.

[20] M. Raza, M. Javaid, and N. Saleem, "Fractional metric dimension of metal-organic frameworks," Main Group Metal Chemistry, vol. 44, no. 1, pp. 92-102, 2021.

[21] M. Raza, D. A. Alrowaili, M. Javaid, and K. Shabbir, "Computing bounds of fractional metric dimension of metal organic graphs," Journal of Chemistry, vol. 2021, Article ID 5539569, 12 pages, 2021.

[22] S. Aisyah, M. I. Utoyo, and L. Susilowati, "On the local fractional metric dimension of corona product graphs," IOP Conference Series: Earth and Environmental Science, Hungarica, vol. 243, 2019.

[23] J.-B. Liu, M. K. Aslam, and M. Javaid, "Local fractional metric dimensions of rotationally symmetric and planar networks," IEEE Access, vol. 8, no. 1, pp. 82404-82420, 2020.

[24] M. Javaid, M. Raza, P. Kumam, and J.-B. Liu, "Sharp bounds of local fractional metric dimensions of connected networks," IEEE Access, vol. 8, no. 2, pp. 172329-172342, 2020.

[25] M. Javaid, M. S. Behzad, U. Farooq, and M. K. Aslam, "Computing sharp bounds for local fractional metric dimensions of cycle related graphs," Computational Journal of Combinatorial Mathematics, vol. 1, pp. 31-75, 2020.

[26] M. A. Johnson, "Browsable structure-activity datasets," in Advances in Molecular Similarity, R. Carbó-Dorca and P. Mezey, Eds., pp. 153-170, JAI Press, Connecticut, 1998. 
[27] M. Johnson, "Structure-activity maps for visualizing the graph variables arising in drug design," Journal of Biopharmaceutical Statistics, vol. 3, pp. 203-236, 1993.

[28] G. Chartrand and P. Zhang, "The theory and applicaitons of resolvability in graphs," Congruent number, vol. 160, no. 1, pp. $47-68,2003$. 